\title{
Temporally, spatially, or lightlike modulated vacua in Lorentz invariant theories
}

\author{
Sven Bjarke Gudnason, ${ }^{1, *}$ Muneto Nitta, ${ }^{1, \dagger}$ Shin Sasaki, ${ }^{2, \$}$ and Ryo Yokokura ${ }^{1, \S}$ \\ ${ }^{1}$ Department of Physics, and Research and Education Center for Natural Sciences, Keio University, \\ Hiyoshi 4-1-1, Yokohama, Kanagawa 223-8521, Japan \\ ${ }^{2}$ Department of Physics, Kitasato University, Sagamihara 252-0373, Japan
}

(Received 12 December 2018; published 20 February 2019)

\begin{abstract}
We study the vacuum structure of a class of Lorentz-invariant field theories where the vacuum expectation values are not constant but are (phase) modulated. The vacua are classified into spatial, temporal, and lightlike modulation types according to the patterns of spontaneous breaking of translational symmetry. The conditions for having temporal or lightlike modulated vacua imply severe constraints on the models. We utilize the notion of generalized Nambu-Goldstone modes which appear in the modulated vacua. Finally, we examine fluctuation modes around these vacua and discuss their dynamics and the absence of ghosts.
\end{abstract}

DOI: 10.1103/PhysRevD.99.045011

\section{INTRODUCTION}

Finding the vacuum structure is one of the most fundamental issues in the course of understanding quantum field theories. The true (false) vacuum in field theories is defined by being the global (a local) minimum of the energy functional (Hamiltonian). For models that consist of a canonical kinetic term (quadratic with second-order spacetime derivative) with a potential $V$, this is given by a constant field configuration determined by a minimum of $V$. In this case, the constant vacuum expectation value (VEV) trivially satisfies the equation of motion and the constant field configurations automatically yield the minimum energy since varying a field costs gradient energy. Contrary to this, the situation can generally become more involved when we consider models that contain quartic or higher-order kinetic terms in addition to the canonical one, but only if not all terms appear in the energy functional with a positive sign. Such models have terms with more than two spacetime derivatives and are called higher-derivative models. In some cases, nonconstant field configurations can be energetically favored and become the vacuum. Depending on the symmetries of the potential, the energy may be

\footnotetext{
*gudnason@keio.jp

nitta@phys-h.keio.ac.jp

shin-s@kitasato-u.ac.jp

${ }^{\S}$ ryokokur@keio.jp
}

Published by the American Physical Society under the terms of the Creative Commons Attribution 4.0 International license. Further distribution of this work must maintain attribution to the author(s) and the published article's title, journal citation, and DOI. Funded by SCOAP ${ }^{3}$. minimized by a field configuration that has nonvanishing derivatives in the vacuum (lowest-energy state). The symmetry that will play an important role in this paper is shift symmetry, which when possessed by the potential allows for the field to be nonconstant. In this situation, the kinetic term and the higher-order derivative terms can be seen as a "potential" for the "velocity" of the field, that is, for a nonvanishing gradient of the field. As mentioned above, if not all terms enter the energy functional with a positive sign, the "velocity" that minimizes the energy may be a nonvanishing constant. Even if the canonical kinetic term has the usual positive sign in the energy functional, a constant VEV may be just a local vacuum, and instead a modulated vacuum becomes the global vacuum. In this paper we will study the situation where the "velocity" is the phase of a complex field, although that is not necessarily the only possibility in general.

In condensed matter physics as opposed to relativistic quantum field theory, the lowest-energy state is not the vacuum but is called the ground state. Modulated ground states were originally discovered in nonrelativistic theories, i.e., theories without Lorentz invariance, in the context of superconductors. In such theories, spatially modulated ground states can easily be found. For example, FuldeFerrell-Larkin-Ovchinnikov (FFLO) states in superconductors were proposed a long time ago [1,2]. For the Fulde-Ferrell (FF) state [1], the phase of a condensate in the ground state is spatially modulated along one spatial direction, while for the Larkin-Ovchinnikov (LO) [2], the amplitude of the condensate is modulated. The LO state is the ground state when an external magnetic field is applied [3] (see also Ref. [4]), while the FF state can be the ground state when a magnetic field penetrating a superconducting 
ring is present (see e.g., Ref. [5]). Recent experiments reveal that these FFLO states are realized in several physical systems [6-9]. In addition to condensed matter physics, the FFLO states appear in a variety of fields. In the context of QCD, spatially modulated chiral condensates of the FF type, called a dual chiral density wave or chiral spiral [10,11], and the LO type, called a real kink crystal [12,13], were contemplated to appear in a certain region of the QCD phase diagram. In the Gross-Neveu or Nambu-Jona Lasino model in $1+1$ dimensions, FFLO states for which both the phase and the amplitude are modulated, called twisted kink crystals $[14,15]$, were discussed at finite temperature and density [16]. The related issues were also discussed in the context of the AdS/CFT correspondence [17-22].

On the other hand, temporal modulations have also been studied recently. The idea of the time crystal [23,24] proposes a time-dependent ground state in which the continuous translational symmetry along the time direction is spontaneously broken to a discrete subgroup. This is an analogue of the ordinary crystalline structure of matter in spatial directions, namely, a conventional crystal. Although the time crystal cannot be realized in thermal equilibrium states [25], it has been reported to be experimentally realized in a nonequilibrium state [26,27]. A diffusive Nambu-Goldstone mode corresponding to spontaneously broken time translation in a time crystal was discussed in Ref. [28]. A temporally modulated state was also studied in QCD [29] and finite-density systems [30].

Further examples of time-dependent vacuum states have also been studied in various contexts. A famous example of such kind of vacuum is ghost condensation [31]. This proposal causes a modification of the long-range gravitational force by means of a Higgs mechanism giving mass to the graviton and is studied mostly in the context of cosmology. In ghost condensation, the VEV depends linearly on time but is not modulated.

In all the examples in the literature that we mentioned so far, i.e., in condensed matter physics or in QCD, Lorentz invariance is either absent or explicitly broken by finite density, temperature, and/or an external magnetic field with the exception of ghost condensation [31]. In ghost condensation, Lorentz invariance is not broken but the kinetic term of the scalar field has the "wrong" sign, thus yielding a ghost.

On the other hand, modulated vacua in Lorentzinvariant setups with a normal kinetic term had not been studied until recently [32,33]. In Refs. [32,33], we realized such a possibility in a simple Lorentz-invariant model with higher-derivative terms, by studying the vacuum structure possessing a nonconstant VEV. ${ }^{1}$ The VEV that we analyzed is a function of a specific

\footnotetext{
${ }^{1}$ In this model, the trivial vacuum at the origin becomes metastable, in contrast to the situation in ghost condensation where it becomes unstable.
}

direction in three-dimensional space. Such kind of vacuum is called spatially modulated.

In this paper, we study modulated vacua in Lorentzinvariant field theories. As a continuation of our previous studies on spatially modulated vacua [32,33], we here focus on temporally and lightlike modulated vacua. Nevertheless, it proves instructive to include the spatially modulated case here so as to put them all on equal footing. A temporally modulated vacuum is characterized by the property that the time derivative of fields develop a VEV. If the solution we are looking for is timelike-due to the Lorentz invariance of the class of theories we are considering-we can always set the spatial derivatives to zero without loss of generality simply by a change of Lorentz frame. Similarly, the lightlike modulation is defined by a VEV for the derivative of fields on the light-cone. A nontrivial problem for timedependent solutions is that the extrema of the Hamiltonian do not necessarily coincide with all solutions to the Lagrangian equation of motion. A subset of the solutions to the Lagrangian equations of motion, as we will show in this paper, do yield extrema of the corresponding Hamiltonian; these are the states we are looking for. In the class of theories we consider in this paper, we will furthermore assume that there is no dependence on the field, $\varphi$, itself in the Lagrangian and that the Lagrangian only depends on the first derivatives of the field, $\partial_{m} \varphi$.

In restricting our discussion to a Lagrangian that only depends (nonlinearly) on the first time derivative of the field, we sidestep the problem of the Ostrogradsky instability and related ghost $[34,35]$. As in the ordinary vacuum in field theories, once the fields develop nontrivial VEVs, some of the symmetries of the Lagrangian are spontaneously broken. In the modulated states in nonrelativistic theories, there appears Nambu-Goldstone (NG) modes associated with broken symmetries [28,36,37]. We will show that there is an analogue of NG modes (generalized NG modes) in our models. The generalized NG modes correspond to the flat direction of the "potential" whose quadratic kinetic term disappears in the Lagrangian for the case of spatial modulation [32]. However, this turns out not to be the case for temporal or lightlike modulation.

In this paper, we study the FF-type (phase) modulation $\varphi \sim \exp (i \omega t)$. One may wonder that this modulation is rather usual in nonrelativistic theories. For instance, (nonlinear) Schrödinger systems, $i \partial_{t} \varphi=\nabla \varphi+V \varphi\left(i \partial_{t} \varphi=\right.$ $\left.\nabla \varphi+g|\varphi|^{2} \varphi\right)$ have states with a time dependence of the above type. Therefore, such a time dependence is not usually called a temporal modulation. Note, however, that this is not the case for Lorentz-invariant (relativistic) theories discussed in this paper.

The organization of this paper is as follows. In the next section, we discuss the general conditions for modulated vacua. We classify the vacua into the spatial, temporal and lightlike types. In Sec. II B 3, we introduce the notion of generalized NG modes and discuss the relation between 
them and the zero modes of the generalized mass matrix. In Sec. III, by focusing on a higher-derivative model, we show an example of the modulated vacua and the generalized NG modes. We also examine the dynamics of the fluctuation modes in the vacua and discuss the absence of ghosts. Section IV is devoted to a summary and discussion.

\section{GENERAL DISCUSSION ON MODULATED VACUA}

Throughout this paper, we consider Lorentz-invariant theories of a single complex scalar field $\varphi$. We require that the theory does not suffer from the Ostrogradsky instability [34,35] which can be caused by fourth or higher order of spacetime derivative terms. For simplicity of constructing models, we assume that theories have shift symmetry of the fields and a global $U(1)$ symmetry:

$$
\varphi \rightarrow \varphi+c, \quad \varphi \rightarrow e^{i \theta} \varphi,
$$

with constant parameters $c$ and $\theta$. The absence of the Ostrogradsky instability then implies that the Lagrangian consists of $\partial_{m} \varphi, \partial_{m} \bar{\varphi}$ only:

$$
\mathcal{L}=\mathcal{L}\left(\partial_{m} \varphi, \partial_{m} \bar{\varphi}\right)
$$

In general, vacua are defined as field configurations that minimize the energy density and they satisfy the equation of motion. The conventional vacua, which are characterized by constant VEVs, trivially satisfy these conditions. However, if we look for vacua where VEVs are not constant but generally spacetime-dependent functions, the conditions are not trivially satisfied. In the following, we write down the general conditions for vacua in this theory and discuss modulated vacua.

\section{A. Conditions for modulated vacua}

Starting from the Lagrangian (2), the equation of motion is

$$
\begin{aligned}
0= & \partial_{m} \frac{\partial \mathcal{L}}{\partial\left(\partial_{m} \varphi\right)}=\frac{\partial^{2} \mathcal{L}}{\partial\left(\partial_{m} \varphi\right) \partial\left(\partial_{n} \varphi\right)} \partial_{m} \partial_{n} \varphi \\
& +\frac{\partial^{2} \mathcal{L}}{\partial\left(\partial_{m} \varphi\right) \partial\left(\partial_{n} \bar{\varphi}\right)} \partial_{m} \partial_{n} \bar{\varphi}, \\
0= & \partial_{m} \frac{\partial \mathcal{L}}{\partial\left(\partial_{m} \bar{\varphi}\right)}=\frac{\partial^{2} \mathcal{L}}{\partial\left(\partial_{m} \bar{\varphi}\right) \partial\left(\partial_{n} \varphi\right)} \partial_{m} \partial_{n} \varphi \\
& +\frac{\partial^{2} \mathcal{L}}{\partial\left(\partial_{m} \bar{\varphi}\right) \partial\left(\partial_{n} \bar{\varphi}\right)} \partial_{m} \partial_{n} \bar{\varphi} .
\end{aligned}
$$

This is expressed in compact form as

$$
\begin{aligned}
\mathbf{L}^{m n}\left(\begin{array}{c}
\partial_{m} \partial_{n} \varphi \\
\partial_{m} \partial_{n} \bar{\varphi}
\end{array}\right)= & \mathbf{L}^{00}\left(\begin{array}{c}
\ddot{\varphi} \\
\ddot{\bar{\varphi}}
\end{array}\right)+\left(\mathbf{L}^{0 i}+\mathbf{L}^{i 0}\right)\left(\begin{array}{c}
\partial_{i} \dot{\varphi} \\
\partial_{i} \dot{\bar{\varphi}}
\end{array}\right) \\
& +\mathbf{L}^{i j}\left(\begin{array}{c}
\partial_{i} \partial_{j} \varphi \\
\partial_{i} \partial_{j} \bar{\varphi}
\end{array}\right)=0 .
\end{aligned}
$$

Here $\dot{\varphi}=\partial_{0} \varphi$ and so on and the $2 \times 2$ matrices $\mathbf{L}^{m n}(m$, $n=0,1,2,3)$ are defined as

$$
\mathbf{L}^{m n} \equiv\left(\begin{array}{cc}
\frac{\partial^{2} \mathcal{L}}{\partial\left(\partial_{m} \bar{\varphi}\right) \partial\left(\partial_{n} \varphi\right)} & \frac{\partial^{2} \mathcal{L}}{\partial\left(\partial_{m} \bar{\varphi}\right) \partial\left(\partial_{n} \bar{\varphi}\right)} \\
\frac{\partial^{2} \mathcal{L}}{\partial\left(\partial_{m} \varphi\right) \partial\left(\partial_{n} \varphi\right)} & \frac{\partial^{2} \mathcal{L}}{\partial\left(\partial_{m} \varphi\right) \partial\left(\partial_{n} \bar{\varphi}\right)}
\end{array}\right) .
$$

Note that only the symmetric part $\mathbf{L}^{(m n)}$ contributes to the equation of motion and $\mathbf{L}^{\dagger 00}=\mathbf{L}^{00}, \quad \mathbf{L}^{\dagger 0 i}=\mathbf{L}^{i 0}$, $\mathbf{L}^{\dagger i j}=\mathbf{L}^{j i}$.

Any global or metastable vacuum of the theory satisfies the equation of motion and it should be a local minimum of the energy functional. The Hamiltonian (energy) density is defined by

$$
\mathcal{H}=\frac{\partial \mathcal{L}}{\partial \dot{\varphi}} \dot{\varphi}+\frac{\partial \mathcal{L}}{\partial \dot{\bar{\varphi}}} \dot{\bar{\varphi}}-\mathcal{L}
$$

The Hamiltonian depends only on the spacetime derivative of fields. Therefore it can be considered as a potential for $\partial_{m} \varphi$. The extremal condition of the energy with respect to $\partial_{m} \varphi$ is then

$$
\begin{aligned}
\frac{\partial \mathcal{H}}{\partial\left(\partial_{m} \varphi\right)}= & \frac{\partial^{2} \mathcal{L}}{\partial \dot{\varphi} \partial\left(\partial_{m} \varphi\right)} \dot{\varphi}+\frac{\partial \mathcal{L}}{\partial \dot{\varphi}} \delta^{m}{ }_{0}+\frac{\partial^{2} \mathcal{L}}{\partial \dot{\bar{\varphi}} \partial\left(\partial_{m} \varphi\right)} \dot{\bar{\varphi}} \\
& -\frac{\partial \mathcal{L}}{\partial\left(\partial_{m} \varphi\right)}=0,
\end{aligned}
$$

and its complex conjugate. More explicitly, we have

$$
\begin{aligned}
\frac{\partial \mathcal{H}}{\partial \dot{\varphi}} & =\frac{\partial^{2} \mathcal{L}}{\partial \dot{\varphi}^{2}} \dot{\varphi}+\frac{\partial^{2} \mathcal{L}}{\partial \dot{\bar{\varphi}} \partial \dot{\varphi}} \dot{\bar{\varphi}}=0 \\
\frac{\partial \mathcal{H}}{\partial\left(\partial_{i} \varphi\right)} & =\frac{\partial^{2} \mathcal{L}}{\partial \dot{\varphi} \partial\left(\partial_{i} \varphi\right)} \dot{\varphi}+\frac{\partial^{2} \mathcal{L}}{\partial \dot{\bar{\varphi}} \partial\left(\partial_{i} \varphi\right)} \dot{\bar{\varphi}}-\frac{\partial \mathcal{L}}{\partial\left(\partial_{i} \varphi\right)}=0 .
\end{aligned}
$$

These together with their complex conjugates are expressed as

$$
\mathbf{L}^{00}\left(\begin{array}{c}
\dot{\varphi} \\
\dot{\bar{\varphi}}
\end{array}\right)=0, \quad \mathbf{L}^{i 0}\left(\begin{array}{c}
\dot{\varphi} \\
\dot{\bar{\varphi}}
\end{array}\right)-\left(\begin{array}{c}
\frac{\partial \mathcal{L}}{\partial\left(\partial_{i} \bar{\varphi}\right)} \\
\frac{\partial \mathcal{L}}{\partial\left(\partial_{i} \varphi\right)}
\end{array}\right)=0 .
$$

One finds that the conventional vacuum configuration which is characterized by a constant VEV trivially satisfies the equation of motion (4) and the extremal condition of the energy density (9). However as we will see in Sec. III, there can be nontrivial solutions to these Eqs. (4), (9) where parts of $\partial_{m} \varphi$ develop nonzero $\operatorname{VEV~}\left\langle\partial_{m} \varphi\right\rangle \neq 0$. 
In order to examine whether a configuration that satisfies Eqs. (4) and (9) is a stable vacuum, we consider the fluctuation $\phi$ in the configuration. We consider a shift in the field $\varphi \rightarrow\langle\varphi\rangle+\phi$, or equivalently $\partial_{m} \varphi \rightarrow\left\langle\partial_{m} \varphi\right\rangle+\partial_{m} \phi$. After the shift in the fields, the Hamiltonian is expanded as

$$
\begin{aligned}
\mathcal{H}\left(\left\langle\partial_{m} \varphi\right\rangle+\partial_{m} \phi,\left\langle\partial_{m} \bar{\varphi}\right\rangle+\partial_{m} \bar{\phi}\right) \\
=\mathcal{H}_{0}+\left.\frac{\partial \mathcal{H}}{\partial\left(\partial_{m} \varphi\right)}\right|_{0} \partial_{m} \phi+\left.\frac{\partial \mathcal{H}}{\partial\left(\partial_{m} \bar{\varphi}\right)}\right|_{0} \partial_{m} \bar{\phi} \\
\quad+\left.\frac{1}{2} \vec{\phi}_{m}^{\dagger} \mathbf{M}^{m n}\right|_{0} \vec{\phi}_{n}+\cdots,
\end{aligned}
$$

where the symbol $\left.\right|_{0}$ means that they are evaluated in the vacuum and we have defined the $2 \times 2$ matrices $\mathbf{M}^{m n}$ and the vector $\vec{\phi}_{m}$ as

$$
\mathbf{M}^{m n} \equiv\left(\begin{array}{cc}
\frac{\partial^{2} \mathcal{H}}{\partial\left(\partial_{m} \bar{\varphi}\right) \partial\left(\partial_{n} \varphi\right)} & \frac{\partial^{2} \mathcal{H}}{\partial\left(\partial_{m} \bar{\varphi}\right) \partial\left(\partial_{n} \bar{\varphi}\right)} \\
\frac{\partial^{2} \mathcal{H}}{\partial\left(\partial_{m} \varphi\right) \partial\left(\partial_{n} \varphi\right)} & \frac{\partial^{2} \mathcal{H}}{\partial\left(\partial_{m} \varphi\right) \partial\left(\partial_{n} \bar{\varphi}\right)}
\end{array}\right), \quad \vec{\phi}_{m} \equiv\left(\begin{array}{c}
\partial_{m} \phi \\
\partial_{m} \bar{\phi}
\end{array}\right)
$$

Note that $\mathbf{M}^{\dagger 00}=\mathbf{M}^{00}, \mathbf{M}^{\dagger 0 i}=\mathbf{M}^{i 0}, \mathbf{M}^{\dagger i j}=\mathbf{M}^{j i}$. The second and the third terms in Eq. (10) vanish $\left.\frac{\partial \mathcal{H}}{\partial\left(\partial_{m} \varphi\right)}\right|_{0}=$ $\left.\frac{\partial \mathcal{H}}{\partial\left(\partial_{m} \bar{\varphi}\right)}\right|_{0}=0$ due to the extremal condition of the energy density (8). The matrices $\mathbf{M}^{m n}$ are expressed by the Lagrangian matrices (5):

$\mathbf{M}^{m n}=\frac{\partial \mathbf{L}^{m n}}{\partial \dot{\varphi}} \dot{\varphi}+\frac{\partial \mathbf{L}^{m n}}{\partial \dot{\bar{\varphi}}} \dot{\bar{\varphi}}+\mathbf{L}^{0 n} \delta^{m}{ }_{0}+\mathbf{L}^{m 0} \delta^{n}{ }_{0}-\mathbf{L}^{m n}$

More explicitly, we have

$$
\begin{aligned}
\mathbf{M}^{00} & =\frac{\partial \mathbf{L}^{00}}{\partial \dot{\varphi}} \dot{\varphi}+\frac{\partial \mathbf{L}^{00}}{\partial \dot{\bar{\varphi}}} \dot{\bar{\varphi}}+\mathbf{L}^{00}, \\
\mathbf{M}^{0 i} & =\frac{\partial \mathbf{L}^{0 i}}{\partial \dot{\varphi}} \dot{\varphi}+\frac{\partial \mathbf{L}^{0 i}}{\partial \dot{\bar{\varphi}}} \dot{\bar{\varphi}}, \\
\mathbf{M}^{i j} & =\frac{\partial \mathbf{L}^{i j}}{\partial \dot{\varphi}} \dot{\varphi}+\frac{\partial \mathbf{L}^{i j}}{\partial \dot{\bar{\varphi}}} \dot{\bar{\varphi}}-\mathbf{L}^{i j} .
\end{aligned}
$$

In order to define the Hessian matrix of the energy functional, we rearrange the expression (10):

$$
\mathcal{H}=\mathcal{H}_{0}+\left.\frac{1}{2} \vec{\phi}_{m}^{\dagger} \mathbf{M}^{m n}\right|_{0} \vec{\phi}_{n}=\mathcal{H}_{0}+\left.\frac{1}{2} \vec{\Phi}^{\dagger} \mathcal{M}\right|_{0} \vec{\Phi}
$$

where we have defined the vector

$$
\vec{\Phi} \equiv\left(\begin{array}{c}
\partial_{0} \phi \\
\partial_{0} \bar{\phi} \\
\partial_{1} \phi \\
\vdots \\
\partial_{3} \bar{\phi}
\end{array}\right)=\left(\begin{array}{c}
\vec{\phi}_{0} \\
\vec{\phi}_{1} \\
\vdots \\
\vec{\phi}_{3}
\end{array}\right),
$$

and the generalized $2 d \times 2 d$ mass matrix

$$
\mathcal{M} \equiv\left(\begin{array}{cccc}
\mathbf{M}^{00} & \mathbf{M}^{01} & \ldots & \mathbf{M}^{03} \\
\mathbf{M}^{10} & \mathbf{M}^{11} & \ldots & \\
\vdots & & \ddots & \\
\mathbf{M}^{30} & \ldots & & \mathbf{M}^{33}
\end{array}\right)
$$

Since $\mathbf{M}^{\dagger m n}=\mathbf{M}^{n m}$, the $2 d \times 2 d=8 \times 8$ matrix $\mathcal{M}$ is a Hermitian Hessian matrix. In order for a vacuum $\left\langle\partial_{m} \varphi\right\rangle \neq 0$ to be stable, the generalized mass matrix needs to be positive semidefinite in the vacuum which we denote $\left.\mathcal{M}\right|_{0} \geq 0$.

Once a vacuum $\left\langle\partial_{\hat{m}} \varphi\right\rangle \neq 0$, for fixed $\hat{m}$, that satisfies Eqs. (4), (8) and $\left.\mathcal{M}\right|_{0} \geq 0$ is found, we consider the dynamical (fluctuation) field $\phi$ around the vacuum. Then the Lagrangian for the dynamical field is

$$
\begin{aligned}
\mathcal{L}= & \mathcal{L}_{0}+\left.\frac{\partial \mathcal{L}}{\partial(\partial \dot{\varphi})}\right|_{0} \partial_{0} \phi+\left.\frac{\partial \mathcal{L}}{\partial(\partial \dot{\bar{\varphi}})}\right|_{0} \partial_{0} \bar{\phi}+\left.\frac{\partial \mathcal{L}}{\partial\left(\partial_{i} \varphi\right)}\right|_{0} \partial_{i} \phi \\
& +\left.\frac{\partial \mathcal{L}}{\partial\left(\partial_{i} \bar{\varphi}\right)}\right|_{0} \partial_{i} \bar{\phi}+\left.\frac{1}{2} \vec{\phi}_{0}^{\dagger} \mathbf{L}^{00}\right|_{0} \vec{\phi}_{0}+\left.\frac{1}{2} \vec{\phi}_{0}^{\dagger} \mathbf{L}^{0 i}\right|_{0} \vec{\phi}_{i} \\
& +\left.\frac{1}{2} \vec{\phi}_{i}^{\dagger} \mathbf{L}^{i 0}\right|_{0} \vec{\phi}_{0}+\left.\frac{1}{2} \vec{\phi}_{i}^{\dagger} \mathbf{L}^{i j}\right|_{0} \vec{\phi}_{j}+\cdots
\end{aligned}
$$

Here $\mathbf{L}^{m n}$ are the matrices defined in Eq. (5) and $\mathcal{L}_{0}$ is a constant.

In the next subsection, we examine nontrivial solutions to the conditions (4) and (9). Among other things, we focus on vacuum configurations where the VEVs are modulated with respect to spacetime coordinates. We first classify the modulated vacua and then clarify the conditions for the vacua.

\section{B. Classification of modulated vacua}

The modulated vacua are classified according to the patterns of spontaneous breaking of the global and Poincaré symmetries. When some components of $\partial_{\hat{m}} \varphi$ develop a nonzero VEV, they break translational symmetry ${ }^{2}$ along the $x^{\hat{m}}$ directions as well as the rotational symmetry in a plane spanned by the $x^{\hat{m}}$ coordinate. The shift and global $U(1)$ symmetries are also spontaneously broken. If the VEV is a

\footnotetext{
${ }^{2}$ Recently, the spontaneous breaking of the translational symmetry in a higher derivative model without the Ostrogradsky instability was discussed [38].
} 
Fulde-Ferrell (FF, the phase modulated) type, i.e., $\left\langle\partial_{\hat{m}} \varphi\right\rangle \propto$ $e^{i c x^{n}}$ with a constant $c$, then the symmetry breaking pattern is classified by the vector generators $P^{\hat{m}}$ associated with the translational symmetry along the $x^{\hat{m}}$ direction into three cases (1) spacelike, (2) timelike and (3) lightlike.

(1) When $P^{\hat{m}}$ is spacelike, the vacuum is called spatially modulated. In this case, we can choose $\hat{m}=1$ without loss of generality. The symmetry is spontaneously broken as $I S O(1,3) \times U(1) \times S \rightarrow I S O(1,2) \rtimes$ $\left[U(1) \times \mathcal{P}^{1}\right]_{\text {diag }}$. Here $S$ is the shift symmetry group, $\mathcal{P}^{1}$ is the translational group generated by $P^{1}$ and diag. stands for the diagonal subgroup corresponding to the simultaneous transformation:

$$
x^{1} \rightarrow x^{1}+a, \quad \varphi \rightarrow e^{-i c a} \varphi,
$$

and $\rtimes$ implies a semidirect product.

(2) When $P^{\hat{m}}$ is timelike, the vacuum is called temporally modulated. In this case, we can choose $\hat{m}=0$ without loss of generality. In this vacuum, the symmetry is spontaneously broken as $\operatorname{ISO}(1,3) \times$ $U(1) \times S \rightarrow I S O(3) \rtimes\left[U(1) \times \mathcal{P}^{0}\right]_{\text {diag }}$ where $I S O(3)$ is the Poincare group in the spatial directions. ${ }^{3}$

(3) On the other hand, when $P^{\hat{m}}$ is lightlike (null), we can choose $P^{\hat{m}}$ to be the light-cone directions $P^{ \pm}=$ $P^{0} \pm P^{1}$. We call the vacuum lightlike modulated. The breaking pattern is $I S O(1,3) \times U(1) \times S \rightarrow$ $I S O(2) \times\left[U(1) \times \mathcal{P}^{ \pm}\right]_{\text {diag }}$.

We will discuss a generalization of the NG modes associated with these symmetry breakings in Sec. II B 3 . In the following, we examine the conditions for each modulated vacuum.

\section{Spatial modulation}

In the case of the spatial modulation, the VEV is characterized by

$$
\langle\dot{\varphi}\rangle=\left\langle\partial_{2} \varphi\right\rangle=\left\langle\partial_{3} \varphi\right\rangle=0, \quad\left\langle\partial_{1} \varphi\right\rangle \neq 0 .
$$

Assuming the ansatz (19), we examine the conditions (4) and (9). By this assumption, we look for static field configurations. We first demand the following conditions:

$$
\left.\frac{\partial \mathcal{L}}{\partial\left(\partial_{i} \varphi\right)}\right|_{0}=0, \quad(i=1,2,3)
$$

Note that $\left.\right|_{0}$ means that the conditions are satisfied in the vacuum for any $x^{i}$. If we impose the above conditions, the energy extremal conditions in Eq. (9) are trivially satisfied. The equation of motion (4) is also trivially satisfied since $\varphi$

\footnotetext{
${ }^{3}$ This kind of symmetry breaking in Lorentz-invariant theories has been discussed in Ref. [39].
}

is $x^{0}$ independent and $\frac{\partial \mathcal{L}}{\partial\left(\partial_{i} \varphi\right)}=0$ for any $x^{i}$. Indeed, for a static field configuration, we have the relation $\mathcal{H}=-\mathcal{L}$ and the condition $\delta \mathcal{H}=0$ automatically implies $\delta \mathcal{L}=0$. In Refs. [32,33] we solved the condition (20) and found a spatially modulated vacuum.

If the theory that we consider is Lorentz invariant, one notices that only the combinations $\dot{\varphi}^{2}, \dot{\varphi} \dot{\bar{\varphi}}$ appear in the Lagrangian:

$$
\mathcal{L} \supset A \dot{\varphi}^{2}, B \dot{\varphi} \dot{\bar{\varphi}},
$$

where $A, B$ are any Lorentz-invariant terms. Then, one can show that the relations

$$
\left.\frac{\partial \mathcal{L}}{\partial \dot{\varphi}}\right|_{\dot{\varphi}=0}=\left.\frac{\partial^{2} \mathcal{L}}{\partial \dot{\varphi} \partial\left(\partial_{i} \varphi\right)}\right|_{\dot{\varphi}=0}=0
$$

always hold for the spatial modulation ansatz (19). From Eq. (13), we therefore find the relations

$$
\left.\mathbf{M}^{00}\right|_{0}=\left.\mathbf{L}^{00}\right|_{0},\left.\quad \mathbf{M}^{i j}\right|_{0}=-\left.\mathbf{L}^{i j}\right|_{0} .
$$

In particular one can show that $\left.\mathbf{L}^{0 i}\right|_{0}=\left.\mathbf{L}^{i 0}\right|_{0}=\left.\mathbf{M}^{0 i}\right|_{0}=0$ for the spatial modulation. The generalized mass matrix is given by

$$
\left.\mathcal{M}\right|_{0}=\left(\begin{array}{c|c}
\left.\mathbf{L}^{00}\right|_{0} & 0 \\
\hline 0 & -\left.\operatorname{diag}\left(\mathbf{L}^{i j}\right)\right|_{0}
\end{array}\right) .
$$

This is completely block diagonal. The explicit expression for $\mathcal{M}$ depends on the models under consideration. For the vacuum (19), we require that $\left.\mathcal{M}\right|_{0}$ is positive semidefinite.

Once a vacuum is found, the Lagrangian for the fluctuation in a spatially modulated vacuum becomes

$$
\mathcal{L}=\mathcal{L}_{0}+\left.\frac{1}{2} \vec{\phi}_{0}^{\dagger} \mathbf{L}^{00}\right|_{0} \vec{\phi}_{0}+\left.\frac{1}{2} \vec{\phi}_{i}^{\dagger} \mathbf{L}^{i j}\right|_{0} \vec{\phi}_{j}+\cdots
$$

The matrices $\left.\mathbf{L}^{m n}\right|_{0}$ govern the kinetic terms of the fluctuation modes. For the spatial modulation, the zero modes of $\left.\mathbf{M}^{m n}\right|_{0}$ coincide with those of $\left.\mathbf{L}^{m n}\right|_{0}$. This issue will be discussed in Sec. II B 3.

\section{Temporal modulation}

The temporal modulation is characterized by the VEV:

$$
\langle\dot{\varphi}\rangle \neq 0, \quad\left\langle\partial_{i} \varphi\right\rangle=0, \quad(i=1,2,3) .
$$

In the case of temporal modulations, it is not always true that extrema of the energy are solutions to the equation of motion. In order to look for a configuration characterized by Eq. (26), we demand the following conditions: 


$$
\left.\mathbf{L}^{00}\right|_{0}=0,\left.\quad \frac{\partial \mathcal{L}}{\partial\left(\partial_{i} \varphi\right)}\right|_{0}=0
$$

Indeed, the second condition in Eq. (27) allows a solution

$$
\partial_{i} \varphi=0
$$

in Lorentz-invariant models. This substantially implies $\left.\mathbf{L}^{0 i}\right|_{0}=0$. Since the solution (28) implies that $\partial_{i} \partial_{j} \varphi=0$ at any points, the conditions (4) and (9) necessary for modulated vacua are now satisfied. By the ansatz (26), we have

$$
\begin{aligned}
\left.\mathbf{M}^{00}\right|_{0} & =\left(\frac{\partial \mathbf{L}^{00}}{\partial \dot{\varphi}} \dot{\varphi}\right)_{0}+\left(\frac{\partial \mathbf{L}^{00}}{\partial \dot{\bar{\varphi}}} \dot{\bar{\varphi}}\right)_{0} \\
\left.\mathbf{M}^{0 i}\right|_{0} & =\left(\frac{\partial \mathbf{L}^{0 i}}{\partial \dot{\varphi}} \dot{\varphi}\right)_{0}+\left(\frac{\partial \mathbf{L}^{0 i}}{\partial \dot{\bar{\varphi}}} \dot{\bar{\varphi}}\right)_{0} \\
\left.\mathbf{M}^{i j}\right|_{0} & =\left(\frac{\partial \mathbf{L}^{i j}}{\partial \dot{\varphi}} \dot{\varphi}\right)_{0}+\left(\frac{\partial \mathbf{L}^{i j}}{\partial \dot{\bar{\varphi}}} \dot{\bar{\varphi}}\right)_{0}-\left.\mathbf{L}^{i j}\right|_{0} .
\end{aligned}
$$

For Lorentz-invariant models, we can show $\left(\frac{\partial \mathbf{L}^{0 i}}{\partial \dot{\varphi}} \dot{\varphi}\right)_{0}=$ $\left(\frac{\partial \mathbf{L}^{0 i}}{\partial \dot{\bar{\varphi}}} \dot{\bar{\varphi}}\right)_{0}=0$, hence $\left.\mathbf{M}^{0 i}\right|_{0}=0$, in the case of temporal modulation but $\left.\mathbf{M}^{00}\right|_{0}$ and $\left.\mathbf{M}^{i j}\right|_{0}$ are not equal to $\left.\mathbf{L}^{00}\right|_{0}$, $\left.\mathbf{L}^{i j}\right|_{0}$, in general. Since $\left.\mathbf{L}^{00}\right|_{0}=0$, the kinetic term of the fluctuation along the time direction vanishes identically. This distinguishes the temporal modulation from the spatial one.

\section{Lightlike modulation}

We define the lightlike modulation as a vacuum characterized by the VEV:

$$
\begin{aligned}
\langle\dot{\varphi}\rangle & \neq 0, \quad\left\langle\partial_{1} \varphi\right\rangle \neq 0, \quad\left\langle\partial_{2} \varphi\right\rangle=\left\langle\partial_{3} \varphi\right\rangle=0, \\
\partial \varphi \cdot \partial \varphi & =\partial \varphi \cdot \partial \bar{\varphi}=0 .
\end{aligned}
$$

Here $\partial \varphi \cdot \partial \varphi=\eta^{m n} \partial_{m} \varphi \partial_{n} \varphi$ and so on. The metric is $\eta_{m n}=\operatorname{diag}(-1,1,1,1)$. In order to look for the configuration (30), we assume

$$
\dot{\varphi}= \pm \partial_{1} \varphi .
$$

If we demand the following conditions:

$$
\begin{gathered}
\left.\mathbf{L}^{00}\right|_{0}=\left.\mathbf{L}^{0 i}\right|_{0}=\left.\mathbf{L}^{i j}\right|_{0}=0, \\
\left.\frac{\partial \mathcal{L}}{\partial\left(\partial_{i} \varphi\right)}\right|_{0}=0,
\end{gathered}
$$

then, both the conditions (4), (9) for modulated vacua are satisfied. However, these conditions are generally too strong and we will find relaxed conditions in a concrete model of lightlike modulation in Sec. III.

\section{GENERALIZED NAMBU-GOLDSTONE MODES}

In the previous section, we made a classification of modulated vacua according to the spontaneous breaking of symmetries. Along with the spontaneous symmetry breakings, we expect that NG modes appear in the spectrum. Particular emphasis is placed on the rotational symmetry. Since the rotations in the $\left(x^{1}, x^{2}\right),\left(x^{1}, x^{3}\right)$ planes are not independent of the translation along the $x^{1}$ direction [40], there are no independent NG modes associated with the spontaneous breaking of the rotational symmetries. Therefore the only relevant part of our interest is $U(1) \times$ $P^{\hat{m}} \rightarrow\left[U(1) \times P^{\hat{m}}\right]_{\text {diag }}$ where $P^{\hat{m}}$ is spacelike, timelike or lightlike. The NG modes associated with these symmetry breakings are well captured by the notion of the generalized NG modes in Ref. [32]. In this section, we discuss a generalization of the NG theorem in our setup. This provides a relation between zero modes of the generalized mass matrix (16) and spontaneous symmetry breaking. Most of the analysis in this section has been already discussed in Ref. [32]. We therefore provide a brief sketch of the notion in the following.

We assume that the energy functional, $\mathcal{H}$, depends on the spacetime derivative of scalar fields only: $\mathcal{H}\left(\varphi_{m}, \bar{\varphi}_{m}\right)$. Here we have defined $\varphi_{m} \equiv \partial_{m} \varphi, \bar{\varphi}_{m} \equiv \partial_{m} \bar{\varphi}$. Vacua are defined as extrema of $\mathcal{H}$ with respect to $\varphi_{m}, \bar{\varphi}_{m}$ :

$$
\frac{\partial \mathcal{H}}{\partial \varphi_{m}}=\frac{\partial \mathcal{H}}{\partial \bar{\varphi}_{m}}=0 .
$$

In these extrema, we assume $\varphi_{m}, \bar{\varphi}_{m}$ have the following VEVs:

$$
\left\langle 0\left|\varphi_{m}\right| 0\right\rangle \equiv v_{m}=\delta^{\hat{m}}{ }_{m} v, \quad\left\langle 0\left|\bar{\varphi}_{m}\right| 0\right\rangle \equiv \bar{v}_{m}=\delta_{m}^{\hat{m}}{ }_{m} \bar{v} .
$$

Here $\hat{m}$ is a fixed spacetime direction. The VEV $v_{m}$ can depend on the spacetime coordinate in general. In that case, the vacuum spontaneously breaks spacetime symmetries. Consider the fluctuations $\phi, \bar{\phi}$ around the VEV. As we have discussed in the previous section, the expansion of the Hamiltonian around the vacua results in $[\vec{\Phi}$ is defined in Eq. (15)]

$\mathcal{H}(v+\phi, \bar{v}+\bar{\phi})=\mathcal{H}(v, \bar{v})+\left.\frac{1}{2} \vec{\Phi}^{\dagger} \mathcal{M}\right|_{0} \vec{\Phi}+\cdots$

In order that the vacua are local minima, we require that $\left.\mathcal{M}\right|_{0}$ is positive semidefinite. The zero modes of $\left.\mathcal{M}\right|_{0}$ correspond to flat directions.

Let us clarify the relation between the zero modes of $\left.\mathcal{M}\right|_{0}$ and symmetries of the theory. The fields $\varphi_{m}, \bar{\varphi}_{m}$ transform as

$$
\left[i \mathcal{Q}^{A}, \varphi_{m}\right]=i\left(T^{A} \varphi\right)_{m}, \quad\left[i \mathcal{Q}^{A}, \bar{\varphi}_{m}\right]=-i\left(T^{A} \bar{\varphi}\right)_{m}
$$


Here $\mathcal{Q}^{A}$ are generators of the symmetry group and $T^{A}$ is a Hermitian matrix representation of the generators. As we mentioned before, a part of $\varphi_{m}, \bar{\varphi}_{m}$ develop VEVs (34). Then the spontaneous breaking of symmetries by modulations is defined if there exist operators $\varphi_{\hat{m}}$ with $v_{\hat{m}} \neq 0$ such that

$$
\left\langle 0\left|\left[i \mathcal{Q}^{A}, \varphi_{\hat{m}}\right]\right| 0\right\rangle=i\left(T^{A} v\right)_{\hat{m}}, \quad\left\langle 0\left|\left[i \mathcal{Q}^{A}, \bar{\varphi}_{\hat{m}}\right]\right| 0\right\rangle=-i\left(T^{A} \bar{v}\right)_{\hat{m}} .
$$

If we define

$$
\left(T^{A} \vec{v}\right)_{\hat{m}} \equiv\left(\begin{array}{c}
\left(T^{A} v\right)_{\hat{m}} \\
-\left(T^{A} \bar{v}\right)_{\hat{m}}
\end{array}\right)
$$

then the symmetry corresponding to $T^{\hat{A}}$ with $T^{\hat{A}} \vec{v}=0$ is preserved in the vacuum while $T^{A^{\prime}}$ such that $T^{A^{\prime}} \vec{v} \neq 0$ is spontaneously broken.

The energy functional $\mathcal{H}$ is invariant under the following transformation:

$\varphi_{m} \rightarrow \varphi_{m}+i \varepsilon^{A}\left(T^{A} \varphi\right)_{m}, \quad \bar{\varphi}_{m} \rightarrow \bar{\varphi}_{m}-i \varepsilon^{A}\left(T^{A} \bar{\varphi}\right)_{m}$,

where $\varepsilon^{A}$ is an infinitesimal parameter. Namely, we have

$$
\delta^{A} \mathcal{H}=\frac{\partial \mathcal{H}}{\partial \varphi_{n}}\left(T^{A} \varphi\right)_{n}-\frac{\partial \mathcal{H}}{\partial \bar{\varphi}_{n}}\left(T^{A} \bar{\varphi}\right)_{n}=0 .
$$

By differentiating this relation with respect to $\varphi_{m}, \bar{\varphi}_{m}$, we obtain

$$
\begin{aligned}
& \frac{\partial^{2} \mathcal{H}}{\partial \varphi_{m} \partial \varphi_{n}}\left(T^{A} \varphi\right)_{n}+\frac{\partial \mathcal{H}}{\partial \varphi_{n}} \frac{\partial}{\partial \varphi_{m}}\left(T^{A} \varphi\right)_{n}-\frac{\partial^{2} \mathcal{H}}{\partial \varphi_{m} \partial \bar{\varphi}_{n}}\left(T^{A} \bar{\varphi}\right)_{n}=0, \\
& \frac{\partial^{2} \mathcal{H}}{\partial \bar{\varphi}_{m} \partial \varphi_{n}}\left(T^{A} \varphi\right)_{n}-\frac{\partial^{2} \mathcal{H}}{\partial \bar{\varphi}_{m} \partial \bar{\varphi}_{n}}\left(T^{A} \bar{\varphi}\right)_{n}-\frac{\partial \mathcal{H}}{\partial \bar{\varphi}_{n}} \frac{\partial}{\partial \bar{\varphi}_{m}}\left(T^{A} \bar{\varphi}\right)_{n}=0 .
\end{aligned}
$$

If we consider these relations in the vacuum, the factor $\partial \mathcal{H} / \partial \varphi_{m}$ vanishes and we find

$$
\left(\begin{array}{cc}
\left.\frac{\partial^{2} \mathcal{H}}{\partial \bar{\varphi}_{m} \partial \varphi_{n}}\right|_{0} & \left.\frac{\partial^{2} \mathcal{H}}{\partial \bar{\varphi}_{m} \partial \bar{\varphi}_{n}}\right|_{0} \\
\left.\frac{\partial^{2} \mathcal{H}}{\partial \varphi_{m} \partial \varphi_{n}}\right|_{0} & \left.\frac{\partial^{2} \mathcal{H}}{\partial \varphi_{m} \partial \bar{\varphi}_{n}}\right|_{0}
\end{array}\right)\left(\begin{array}{c}
\left(T^{A} v\right)_{n} \\
-\left(T^{A} \bar{v}\right)_{n}
\end{array}\right)=0 .
$$

This results in the following relation:

$$
\left.\mathbf{M}^{m n}\right|_{0}\left(T^{A} \vec{v}\right)_{n}=0,
$$

or equivalently

$$
\left.\mathcal{M}\right|_{0}\left(T^{A} \vec{V}\right)=0
$$

Here we have defined $\langle 0|\vec{\Phi}| 0\rangle=\vec{V}$ where $\vec{\Phi}$ is defined in Eq. (15). If $T^{A} \vec{V} \neq 0$, namely, $T^{A}=T^{A^{\prime}}$ is a broken generator, this is an eigenvector of the generalized mass matrix $\left.\mathcal{M}\right|_{0}$ associated with zero eigenvalue. ${ }^{4}$ We call these zero modes $T^{A} \vec{V}$ the generalized NG modes. Note that since only parts of the component in $\vec{V}$ are nonzero, i.e.,

$$
\vec{V}=\left(\begin{array}{c}
0 \\
\vdots \\
\left(T^{A} \vec{v}\right)_{\hat{m}} \\
\vdots \\
0
\end{array}\right),
$$

only the subsector that corresponds to the nonzero VEV directions $\hat{m}$ are relevant to specify the zero modes.

\section{HIGHER DERIVATIVE SCALAR MODEL}

In this section, we demonstrate the existence of modulated vacua by focusing on a concrete model with Lorentz invariance, and study fluctuations in each case according to the classification of spatial, temporal and lightlike modulation.

\section{A. Model}

The model that we will consider here, is based on the global stability considerations presented in Ref. [32], which we will briefly review. We consider a Lagrangian density which only depends on $\partial_{m} \varphi$ and its complex conjugate, $\mathcal{L}\left(\partial_{m} \varphi, \partial_{m} \bar{\varphi}\right)$ and it contains a finite power of the derivative term, $|\partial \varphi|^{2}=\partial_{m} \varphi \partial^{m} \bar{\varphi}$, hence the highest power of the derivative takes the form:

$$
\mathcal{L} \supset \mp|\partial \varphi|^{2 N}=\mp\left(-|\dot{\varphi}|^{2}+|\nabla \varphi|^{2}\right)^{N},
$$

with $N \in \mathbb{Z}_{>0}$ being a positive integer. The spacetime index $m$ has been contracted by the inverse metric $\eta^{m n}=$ $\operatorname{diag}(-1,1,1,1)$ yielding the relative minus sign in the last expression. $\dot{\varphi}$ is the time derivative of $\varphi$ and $\nabla \varphi$ is the spatial gradient vector of $\varphi$. Let us now construct the Hamiltonian by performing a Legendre transformation

$$
\pi=\frac{\partial \mathcal{L}}{\partial \dot{\varphi}} \supset \mp(-1)^{N} N|\partial \varphi|^{2 N-2} \dot{\bar{\varphi}},
$$

yielding

$$
\mathcal{H}=\pi \dot{\varphi}+\bar{\pi} \dot{\bar{\varphi}}-\mathcal{L} \supset \mp(-1)^{N}(2 N-1)|\dot{\varphi}|^{2 N} \pm|\nabla \varphi|^{2 N} .
$$

\footnotetext{
${ }^{4}$ Note that this relation never implies that all the zero modes of $\left.\mathcal{M}\right|_{0}$ are given by $T^{A} \vec{V}$ but the inverse is true. Namely if $T^{A} \vec{V} \neq 0$ exists, it should be a zero mode of $\left.\mathcal{M}\right|_{0}$.
} 
The global stability of the vacuum in this model is given provided that there is no runaway direction. Considering first the limit of $|\nabla \varphi|^{2} \rightarrow \infty$, we can conclude that we must choose the upper sign. Similarly we consider the limit of $|\dot{\varphi}|^{2} \rightarrow \infty$. Since we have chosen the upper sign, we see from the above Hamiltonian that the largest power of the derivative terms, $N$, must be odd. $N=1$ is just the canonical kinetic term. Hence, the simplest nontrivial example with global stability is $N=3$.

We will here consider a higher-derivative scalar field model of the sixth order, corresponding to $N=3$. The most general Lagrangian density of the Lorentz-invariant scalar field theory with $N=3$ that is compatible with the symmetries (1) and the absence of the Ostrogradsky instability is given by

$$
\begin{aligned}
\mathcal{L}= & -k \partial \varphi \cdot \partial \bar{\varphi}+\lambda(\partial \varphi \cdot \partial \varphi)(\partial \bar{\varphi} \cdot \partial \bar{\varphi})+\mu(\partial \varphi \cdot \partial \bar{\varphi})^{2} \\
& +\alpha(\partial \varphi \cdot \partial \bar{\varphi})(\partial \varphi \cdot \partial \varphi)(\partial \bar{\varphi} \cdot \partial \bar{\varphi})+\beta(\partial \varphi \cdot \partial \bar{\varphi})^{3} .
\end{aligned}
$$

The first term corresponds to the canonical kinetic term and the other terms are higher-derivative corrections. The parameters $k, \lambda, \mu, \alpha, \beta$ are constants. When $\beta=\mu=0$, the model reduces to the one studied in Ref. [32]. ${ }^{5}$ The energy density is

$$
\begin{aligned}
\mathcal{H}= & 2 k|\dot{\varphi}|^{2}-4 \mu|\dot{\varphi}|^{2}(\partial \varphi \cdot \partial \bar{\varphi})-2 \alpha|\dot{\varphi}|^{2}(\partial \varphi \cdot \partial \varphi)(\partial \bar{\varphi} \cdot \partial \bar{\varphi}) \\
& -6 \beta|\dot{\varphi}|^{2}(\partial \varphi \cdot \partial \bar{\varphi})^{2}-2 \lambda \dot{\varphi}^{2}(\partial \bar{\varphi} \cdot \partial \bar{\varphi})-2 \lambda \dot{\bar{\varphi}}(\partial \varphi \cdot \partial \varphi) \\
& -2 \alpha \dot{\varphi}^{2}(\partial \varphi \cdot \partial \bar{\varphi})(\partial \bar{\varphi} \cdot \partial \bar{\varphi})-2 \alpha \dot{\bar{\varphi}}(\partial \varphi \cdot \partial \bar{\varphi})(\partial \varphi \cdot \partial \varphi) \\
& +k(\partial \varphi \cdot \partial \bar{\varphi})-\lambda(\partial \varphi \cdot \partial \varphi)(\partial \bar{\varphi} \cdot \partial \bar{\varphi})-\mu(\partial \varphi \cdot \partial \bar{\varphi})^{2} \\
& -\alpha(\partial \varphi \cdot \partial \bar{\varphi})(\partial \varphi \cdot \partial \varphi)(\partial \bar{\varphi} \cdot \partial \bar{\varphi})-\beta(\partial \varphi \cdot \partial \bar{\varphi})^{3} \cdot
\end{aligned}
$$

In order to examine the conditions (4), (9) for modulated vacua, we evaluate the matrices $\mathbf{L}^{m n}$. For the model (49), we have

$$
\mathbf{L}^{m n}=\left(\begin{array}{cc}
\mathbf{L}_{\bar{\varphi} \varphi}^{m n} & \mathbf{L}_{\bar{\varphi} \bar{\varphi}}^{m n} \\
\mathbf{L}_{\varphi \varphi}^{m n} & \mathbf{L}_{\varphi \bar{\varphi}}^{m n}
\end{array}\right),
$$

where each component is calculated as

$$
\begin{aligned}
\mathbf{L}_{\bar{\varphi} \varphi}^{m n} \equiv & -k \eta^{m n}+\alpha \eta^{m n}(\partial \varphi \cdot \partial \varphi)(\partial \bar{\varphi} \cdot \partial \bar{\varphi})+2 \alpha \partial^{m} \bar{\varphi} \partial^{n} \bar{\varphi}(\partial \varphi \cdot \partial \varphi)+2 \alpha \partial^{m} \varphi \partial^{n} \varphi(\partial \bar{\varphi} \cdot \partial \bar{\varphi}) \\
& +4(\lambda+\alpha \partial \varphi \cdot \partial \bar{\varphi}) \partial^{m} \bar{\varphi} \partial^{n} \varphi+2 \mu\left\{\partial^{m} \varphi \partial^{n} \bar{\varphi}+\eta^{m n}(\partial \varphi \cdot \partial \bar{\varphi})\right\}+3 \beta(\partial \varphi \cdot \partial \bar{\varphi})\left\{2 \partial^{m} \varphi \partial^{n} \bar{\varphi}+\eta^{m n}(\partial \varphi \cdot \partial \bar{\varphi})\right\}, \\
\mathbf{L}_{\bar{\varphi} \bar{\varphi}}^{m n} \equiv & 2 \alpha(\partial \varphi \cdot \partial \varphi)\left(\partial^{m} \bar{\varphi} \partial^{n} \varphi+\partial^{m} \varphi \partial^{n} \bar{\varphi}\right)+2 \eta^{m n}(\lambda+\alpha \partial \varphi \cdot \partial \bar{\varphi})(\partial \varphi \cdot \partial \varphi)+2 \mu \partial^{m} \varphi \partial^{n} \varphi+6 \beta(\partial \varphi \cdot \partial \bar{\varphi}) \partial^{m} \varphi \partial^{n} \varphi, \\
\mathbf{L}_{\varphi \varphi}^{m n} \equiv & 2 \alpha(\partial \bar{\varphi} \cdot \partial \bar{\varphi})\left(\partial^{m} \varphi \partial^{n} \bar{\varphi}+\partial^{m} \bar{\varphi} \partial^{n} \varphi\right)+2 \eta^{m n}(\lambda+\alpha \partial \varphi \cdot \partial \bar{\varphi})(\partial \bar{\varphi} \cdot \partial \bar{\varphi})+2 \mu \partial^{m} \bar{\varphi} \partial^{n} \bar{\varphi}+6 \beta(\partial \varphi \cdot \partial \bar{\varphi}) \partial^{m} \bar{\varphi} \partial^{n} \bar{\varphi}, \\
\mathbf{L}_{\varphi \bar{\varphi}}^{m n} \equiv & -k \eta^{m n}+\alpha \eta^{m n}(\partial \varphi \cdot \partial \varphi)(\partial \bar{\varphi} \cdot \partial \bar{\varphi})+2 \alpha \partial^{m} \varphi \partial^{n} \varphi(\partial \bar{\varphi} \cdot \partial \bar{\varphi})+2 \alpha \partial^{m} \bar{\varphi} \partial^{n} \bar{\varphi}(\partial \varphi \cdot \partial \varphi) \\
& +4(\lambda+\alpha \partial \varphi \cdot \partial \bar{\varphi}) \partial^{m} \varphi \partial^{n} \bar{\varphi}+2 \mu\left\{\partial^{m} \bar{\varphi} \partial^{n} \varphi+\eta^{m n}(\partial \varphi \cdot \partial \bar{\varphi})\right\}+3 \beta(\partial \varphi \cdot \partial \bar{\varphi})\left\{2 \partial^{m} \bar{\varphi} \partial^{n} \varphi+\eta^{m n}(\partial \varphi \cdot \partial \bar{\varphi})\right\} .
\end{aligned}
$$

Then, it is straightforward to calculate the other relevant quantities, such as $\frac{\partial \mathbf{L}^{m n}}{\partial \dot{\varphi}}$ and its complex conjugate:

$$
\begin{aligned}
\frac{\partial \mathbf{L}_{\overline{\bar{\varphi}} \varphi}^{m n}}{\partial \dot{\varphi}}= & -2 \alpha \eta^{m n} \dot{\varphi}(\partial \bar{\varphi} \cdot \partial \bar{\varphi})-4 \alpha \dot{\varphi} \partial^{m} \bar{\varphi} \partial^{n} \bar{\varphi}+2 \alpha(\partial \bar{\varphi} \cdot \partial \bar{\varphi})\left(\eta^{m 0} \partial^{n} \varphi+\partial^{m} \varphi \eta^{n 0}\right)-4 \alpha \dot{\bar{\varphi}} \partial^{m} \bar{\varphi} \partial^{n} \varphi+4(\lambda+\alpha \partial \varphi \cdot \partial \bar{\varphi}) \partial^{m} \bar{\varphi} \eta^{n 0} \\
& +2 \mu\left(\eta^{m 0} \partial^{n} \bar{\varphi}-\eta^{m n} \dot{\bar{\varphi}}\right)-3 \beta \dot{\bar{\varphi}}\left\{2 \partial^{m} \varphi \partial^{n} \bar{\varphi}+\eta^{m n}(\partial \varphi \cdot \partial \bar{\varphi})\right\}+3 \beta(\partial \varphi \cdot \partial \bar{\varphi})\left\{2 \eta^{m 0} \partial^{n} \bar{\varphi}-\eta^{m n} \dot{\bar{\varphi}}\right\} \\
\frac{\partial \mathbf{L}_{\bar{\varphi} \bar{\varphi}}^{m n}}{\partial \dot{\varphi}}= & -4 \alpha \dot{\varphi}\left(\partial^{m} \bar{\varphi} \partial^{n} \varphi+\partial^{m} \varphi \partial^{n} \bar{\varphi}\right)+2 \alpha(\partial \varphi \cdot \partial \bar{\varphi})\left(\partial^{m} \bar{\varphi} \eta^{n 0}+\eta^{m 0} \partial^{n} \bar{\varphi}\right)-2 \alpha \eta^{m n} \dot{\bar{\varphi}} \partial \varphi \cdot \partial \bar{\varphi}-4 \eta^{m n} \dot{\varphi}(\lambda+\alpha \partial \varphi \cdot \partial \bar{\varphi}) \\
& +2 \mu\left(\eta^{m 0} \partial^{n} \varphi+\eta^{n 0} \partial^{m} \varphi\right)-6 \beta \dot{\bar{\varphi}} \partial^{m} \varphi \partial^{n} \varphi+6 \beta(\partial \varphi \cdot \partial \bar{\varphi})\left(\eta^{m 0} \partial^{n} \varphi+\eta^{n 0} \partial^{m} \varphi\right) \\
& \frac{\partial \mathbf{L}_{\varphi \varphi}^{m n}}{\partial \dot{\varphi}}=2 \alpha(\partial \bar{\varphi} \cdot \partial \bar{\varphi})\left(\eta^{m 0} \partial^{n} \bar{\varphi}+\partial^{m} \bar{\varphi} \eta^{n 0}\right)-2 \alpha \eta^{m n} \dot{\bar{\varphi}}(\partial \bar{\varphi} \cdot \partial \bar{\varphi})-6 \beta \dot{\bar{\varphi}} \partial^{m} \bar{\varphi} \partial^{n} \bar{\varphi} \\
\frac{\partial \mathbf{L}_{\varphi \bar{\varphi}}^{m n}=}{\partial \dot{\varphi}}= & -2 \alpha \eta^{m n} \dot{\varphi}(\partial \bar{\varphi} \cdot \partial \bar{\varphi})-4 \alpha \dot{\varphi} \partial^{m} \bar{\varphi} \partial^{n} \bar{\varphi}+2 \alpha(\partial \bar{\varphi} \cdot \partial \bar{\varphi})\left(\eta^{m 0} \partial^{n} \varphi+\partial^{m} \varphi \eta^{n 0}\right)-4 \alpha \dot{\bar{\varphi}} \partial^{m} \varphi \partial^{n} \bar{\varphi}+4(\lambda+\alpha \partial \varphi \cdot \partial \bar{\varphi}) \eta^{m 0} \partial^{n} \bar{\varphi} \\
& +2 \mu\left(\eta^{n 0} \partial^{m} \bar{\varphi}-\eta^{m n} \dot{\bar{\varphi}}\right)-3 \beta \dot{\bar{\varphi}}\left\{2 \partial^{m} \bar{\varphi} \partial^{n} \varphi+\eta^{m n}(\partial \varphi \cdot \partial \bar{\varphi})\right\}+6 \beta(\partial \varphi \cdot \partial \bar{\varphi})\left(2 \eta^{n 0} \partial^{m} \bar{\varphi}-\eta^{m n} \dot{\bar{\varphi}}\right)
\end{aligned}
$$

\footnotetext{
${ }^{5}$ This limit is also the bosonic part of the supersymmetric model studied in Ref. [33].
} 


$$
\begin{aligned}
\frac{\partial \mathbf{L}_{\bar{\varphi} \varphi}^{m n}}{\partial \dot{\bar{\varphi}}}= & -2 \alpha \eta^{m n} \dot{\bar{\varphi}}(\partial \varphi \cdot \partial \varphi)+2 \alpha(\partial \varphi \cdot \partial \varphi)\left(\eta^{m 0} \partial^{n} \bar{\varphi}+\partial^{m} \bar{\varphi} \eta^{n 0}\right)-4 \alpha \dot{\bar{\varphi}} \partial^{m} \varphi \partial^{n} \varphi-4 \alpha \dot{\varphi} \partial^{m} \bar{\varphi} \partial^{n} \varphi \\
& +4(\lambda+\alpha \partial \varphi \cdot \partial \bar{\varphi}) \partial^{n} \varphi \eta^{m 0}+2 \mu\left(\eta^{n 0} \partial^{m} \varphi-\eta^{m n} \dot{\varphi}\right)-3 \beta \dot{\varphi}\left\{2 \partial^{m} \varphi \partial^{n} \bar{\varphi}+\eta^{m n}(\partial \varphi \cdot \partial \bar{\varphi})\right\} \\
& +3 \beta(\partial \varphi \cdot \partial \bar{\varphi})\left(2 \eta^{n 0} \partial^{m} \varphi-\eta^{m n} \dot{\varphi}\right), \\
& \frac{\partial \mathbf{L}_{\bar{\varphi} \bar{\varphi}}^{m n}}{\partial \dot{\bar{\varphi}}}=2 \alpha(\partial \varphi \cdot \partial \varphi)\left(\eta^{m 0} \partial^{n} \varphi+\partial^{m} \varphi \eta^{n 0}\right)-2 \alpha \eta^{m n} \dot{\varphi}(\partial \varphi \cdot \partial \varphi)-6 \beta \dot{\varphi} \partial^{m} \varphi \partial^{n} \varphi
\end{aligned}
$$

$$
\begin{aligned}
\frac{\partial \mathbf{L}_{\varphi \varphi}^{m n}}{\partial \dot{\bar{\varphi}}}= & -4 \alpha \dot{\bar{\varphi}}\left(\partial^{m} \varphi \partial^{n} \bar{\varphi}+\partial^{m} \bar{\varphi} \partial^{n} \varphi\right)+2 \alpha(\partial \bar{\varphi} \cdot \partial \bar{\varphi})\left(\partial^{m} \varphi \eta^{n 0}+\eta^{m 0} \partial^{n} \varphi\right)-2 \alpha \eta^{m n} \dot{\varphi}(\partial \bar{\varphi} \cdot \partial \bar{\varphi})-4 \eta^{m n} \dot{\bar{\varphi}}(\lambda+\alpha \partial \varphi \cdot \partial \bar{\varphi}) \\
& +2 \mu\left(\eta^{m 0} \partial^{n} \bar{\varphi}+\eta^{n 0} \partial^{m} \bar{\varphi}\right)-6 \beta \dot{\varphi} \partial^{m} \bar{\varphi} \partial^{n} \bar{\varphi}+6 \beta(\partial \varphi \cdot \partial \bar{\varphi})\left(\eta^{m 0} \partial^{n} \bar{\varphi}+\eta^{n 0} \partial^{m} \bar{\varphi}\right), \\
\frac{\partial \mathbf{L}_{\varphi \bar{\varphi}}^{m n}}{\partial \dot{\bar{\varphi}}}= & -2 \alpha \eta^{m n} \dot{\bar{\varphi}} \partial \varphi \cdot \partial \varphi+2 \alpha(\partial \varphi \cdot \partial \varphi)\left(\eta^{m 0} \partial^{n} \bar{\varphi}+\partial^{m} \bar{\varphi} \eta^{n 0}\right)-4 \alpha \dot{\bar{\varphi}} \partial^{m} \varphi \partial^{n} \varphi-4 \alpha \dot{\varphi} \partial^{m} \varphi \partial^{n} \bar{\varphi}+4(\lambda+\alpha \partial \varphi \cdot \partial \bar{\varphi}) \partial^{m} \varphi \eta^{n 0} \\
& +2 \mu\left(\eta^{m 0} \partial^{n} \varphi-\eta^{m n} \dot{\varphi}\right)-3 \beta \dot{\varphi}\left\{2 \partial^{m} \bar{\varphi} \partial^{n} \varphi+\eta^{m n}(\partial \varphi \cdot \partial \bar{\varphi})\right\}+3 \beta(\partial \varphi \cdot \partial \bar{\varphi})\left(2 \eta^{m 0} \partial^{n} \varphi-\eta^{m n} \dot{\varphi}\right) .
\end{aligned}
$$

We also have

$$
\begin{aligned}
& \frac{\partial \mathcal{L}}{\partial\left(\partial_{i} \varphi\right)}=\left\{-k+\alpha(\partial \varphi \cdot \partial \varphi)(\partial \bar{\varphi} \cdot \partial \bar{\varphi})+2 \mu(\partial \varphi \cdot \partial \bar{\varphi})+3 \beta(\partial \varphi \cdot \partial \bar{\varphi})^{2}\right\} \partial^{i} \bar{\varphi}+2(\lambda+\alpha \partial \varphi \cdot \partial \bar{\varphi})(\partial \bar{\varphi} \cdot \partial \bar{\varphi}) \partial^{i} \varphi \\
& \frac{\partial \mathcal{L}}{\partial\left(\partial_{i} \bar{\varphi}\right)}=\left\{-k+\alpha(\partial \varphi \cdot \partial \varphi)(\partial \bar{\varphi} \cdot \partial \bar{\varphi})+2 \mu(\partial \varphi \cdot \partial \bar{\varphi})+3 \beta(\partial \varphi \cdot \partial \bar{\varphi})^{2}\right\} \partial^{i} \varphi+2(\lambda+\alpha \partial \varphi \cdot \partial \bar{\varphi})(\partial \varphi \cdot \partial \varphi) \partial^{i} \bar{\varphi}
\end{aligned}
$$

Now that we have all the ingredients to examine the conditions (4), (9), we will study the spatial, temporal and lightlike modulations in turn.

\section{B. Spatial modulation}

We first consider the spatially modulated vacuum in the model. As we will show in the following, the results in this subsection are essentially the same with the ones in Ref. [32], but Lagrangian here is more general. In order to make the paper be self-contained, we rederive the results in the more general setup (49).
For the spatial modulation, we employ the ansatz

$$
\langle\varphi\rangle=\varphi_{0} e^{i c x^{1}}
$$

where $\varphi_{0}, c$ are real constants and the VEV is of the FFtype in the $x^{1}$ direction. Following the general procedure discussed in Sec. II B, we examine the spatially modulated vacuum. The ansatz implies

$$
\langle\dot{\varphi}\rangle=\left\langle\partial_{2} \varphi\right\rangle=\left\langle\partial_{3} \varphi\right\rangle=0, \quad\left\langle\partial_{1} \varphi\right\rangle=i c \varphi_{0} e^{i c x^{1}} \neq 0
$$

Then, we have

$$
\frac{\partial \mathcal{L}}{\partial\left(\partial_{i} \varphi\right)}=\left[-k+\alpha\left|\partial_{1} \varphi\right|^{4}+2 \mu\left|\partial_{1} \varphi\right|^{2}+3 \beta\left|\partial_{1} \varphi\right|^{4}\right] \delta_{1}^{i} \partial_{1} \bar{\varphi}+2\left(\lambda+\alpha\left|\partial_{1} \varphi\right|^{2}\right)\left(\partial_{1} \bar{\varphi}\right)^{2} \delta_{1}^{i} \partial_{1} \varphi .
$$

We first study the condition (9). As discussed in Sec. II B, the nontrivial conditions boil down to $\frac{\partial \mathcal{L}}{\partial\left(\partial_{i} \varphi\right)}=0$. The conditions for $i=2,3$ in Eq. (20) are automatically satisfied. On the other hand, for $i=1$, we have

$$
\begin{aligned}
0=\frac{\partial \mathcal{L}}{\partial\left(\partial_{1} \varphi\right)} & =\left\{-k+\alpha|\partial \varphi|^{4}+2 \mu\left|\partial_{1} \varphi\right|^{2}+3 \beta|\partial \varphi|^{4}\right\} \partial_{1} \bar{\varphi}+2\left(\lambda+\alpha|\partial \varphi|^{2}\right)\left|\partial_{1} \varphi\right|^{2} \partial_{1} \bar{\varphi} \\
& =\partial_{1} \bar{\varphi}\left[-k+2(\mu+\lambda)\left|\partial_{1} \varphi\right|^{2}+3(\alpha+\beta)\left|\partial_{1} \varphi\right|^{4}\right] .
\end{aligned}
$$

This determines the amplitude of the VEV:

$$
\left|\partial_{1} \varphi\right|^{2}=\left|c \varphi_{0}\right|^{2}=\frac{-(\lambda+\mu) \pm \sqrt{(\lambda+\mu)^{2}+3 k(\alpha+\beta)}}{3(\alpha+\beta)}
$$



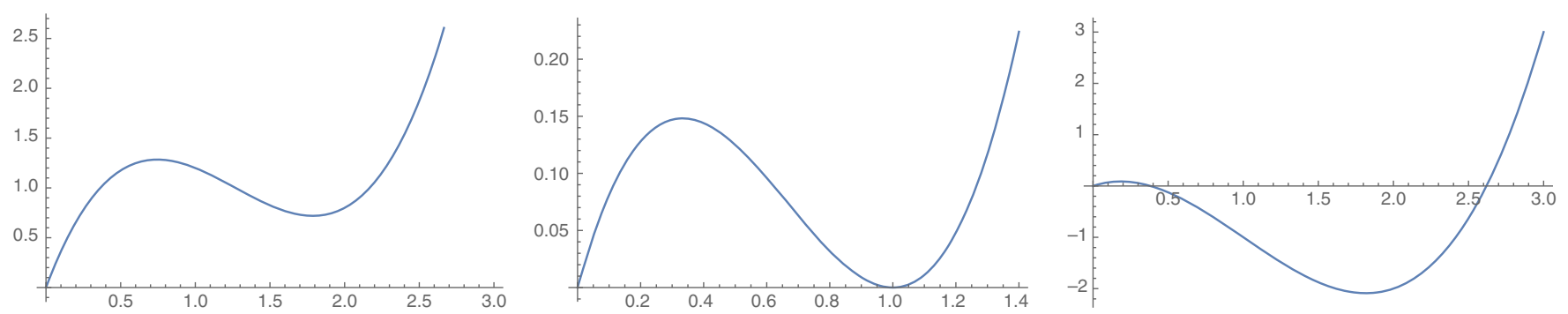

FIG. 1. The energy density as a function of $X=\left|\partial_{1} \varphi\right|^{2}$. The vertical axis represents the energy density while the horizontal axis represents $X$. The parameters are: left (metastable): $k=4, \lambda+\mu=3.8, \alpha+\beta=-1$, middle (degenerate): $k=1, \lambda+\mu=2$, $\alpha+\beta=-1$, right (global): $k=1, \lambda+\mu=3, \alpha+\beta=-1$.

Next, we analyze the other condition, i.e., Eq. (4). The matrices $\mathbf{L}^{m n}$ for the spatial modulation are calculated as

$$
\begin{aligned}
& \mathbf{L}^{00}=\left(\begin{array}{c|c}
k-2 \beta\left|\partial_{1} \varphi\right|^{2}-(\alpha+3 \beta)\left|\partial_{1} \varphi\right|^{4} & -2\left(\partial_{1} \varphi\right)^{2}\left(\lambda+\alpha\left|\partial_{1} \varphi\right|^{2}\right) \\
\hline-2\left(\partial_{1} \bar{\varphi}\right)^{2}\left(\lambda+\alpha\left|\partial_{1} \varphi\right|^{2}\right) & k-2 \beta\left|\partial_{1} \varphi\right|^{2}-(\alpha+3 \beta)\left|\partial_{1} \varphi\right|^{4}
\end{array}\right), \\
& \mathbf{L}^{11}=\left(\begin{array}{c|c}
-k+4(\lambda+\mu)\left|\partial_{1} \varphi\right|^{2}+9(\alpha+\beta)\left|\partial_{1} \varphi\right|^{4} & 2\left(\partial_{1} \varphi\right)^{2}\left\{(\lambda+\mu)+3(\alpha+\beta)\left|\partial_{1} \varphi\right|^{2}\right\} \\
\hline 2\left(\partial_{1} \bar{\varphi}\right)^{2}\left\{(\lambda+\mu)+3(\alpha+\beta)\left|\partial_{1} \varphi\right|^{2}\right\} & -k+4(\lambda+\mu)\left|\partial_{1} \varphi\right|^{2}+9(\alpha+\beta)\left|\partial_{1} \varphi\right|^{4}
\end{array}\right), \\
& \mathbf{L}^{22}=\mathbf{L}^{33}=\left(\begin{array}{c|c}
-k+2 \mu\left|\partial_{1} \varphi\right|^{2}+(\alpha+3 \beta)\left|\partial_{1} \varphi\right|^{4} & 2\left(\partial_{1} \varphi\right)^{2}\left(\lambda+\alpha\left|\partial_{1} \varphi\right|^{2}\right) \\
\hline 2\left(\partial_{1} \bar{\varphi}\right)^{2}\left(\lambda+\alpha\left|\partial_{1} \varphi\right|^{2}\right) & -k+2 \mu\left|\partial_{1} \varphi\right|^{2}+(\alpha+3 \beta)\left|\partial_{1} \varphi\right|^{4}
\end{array}\right),
\end{aligned}
$$

and the others vanish. One immediately finds that the only nontrivial condition comes from the $(i, j)=(1,1)$ component of the third term in Eq. (4). However, the term should vanish from the general discussion. Let us confirm this fact. Although $\mathbf{L}^{11} \neq 0$, we have

$$
\mathbf{L}^{11}\left(\begin{array}{c}
\partial_{1}^{2} \varphi \\
\partial_{1}^{2} \bar{\varphi}
\end{array}\right)=i c\left(\begin{array}{c}
\partial_{1} \varphi\left[-k+2(\lambda+\mu)\left|\partial_{1} \varphi\right|^{2}+3(\alpha+\beta)\left|\partial_{1} \varphi\right|^{4}\right] \\
-\partial_{1} \bar{\varphi}\left[-k+2(\lambda+\mu)\left|\partial_{1} \varphi\right|^{2}+3(\alpha+\beta)\left|\partial_{1} \varphi\right|^{4}\right]
\end{array}\right)
$$

This vanishes due to the condition (68) as expected.

\section{Global aspects of the potential and stability of the vacuum}

Although we have confirmed that the spatially modulated configuration (65) is a solution to the equation of motion and the energy extremum condition, it is still unclear whether the configuration is a (global or local) minimum of the energy functional. As we will show below, this holds true in a specific region of the parameters $k, \alpha, \beta$, $\lambda, \mu$.

We first study the global stability in the $\partial_{1} \varphi$ direction. The energy density for the ansatz (65) is

$$
\mathcal{E}_{\mathrm{sp}}=k\left|\partial_{1} \varphi\right|^{2}-(\lambda+\mu)\left|\partial_{1} \varphi\right|^{4}-(\alpha+\beta)\left|\partial_{1} \varphi\right|^{6}
$$

This is a function of $X \equiv\left|\partial_{1} \varphi\right|^{2} \geq 0: y=k X-(\lambda+\mu) X^{2}-$ $(\alpha+\beta) X^{3}$. From this expression the condition for the existence of a local minimum is determined by the discriminant condition of $y^{\prime}=k-2(\lambda+\mu) X-3(\alpha+\beta) X^{2}=0$ :

$$
\mathbf{D}=4\left\{(\lambda+\mu)^{2}+3 k(\alpha+\beta)\right\}>0 .
$$

This is the condition that the energy has a local minimum at $X \neq 0$. When these conditions are satisfied, one finds that the solution (69) is a local minimum of the energy. Since the energy is given in the form

$\mathcal{E}_{\mathrm{sp}}=X\left[-(\alpha+\beta) X^{2}-(\lambda+\mu) X+k\right], \quad X \equiv\left|\partial_{1} \varphi\right|^{2}$,

one finds that if $f(X) \equiv-(\alpha+\beta) X^{2}-(\lambda+\mu) X+k=0$ has no solution, then, the local minimum (69) is metastable. If $f(X)=0$ has one solution, then the vacuum (69) is degenerate with the trivial vacuum $X=0$. If $f(X)=0$ has two solutions, then (69) becomes the global minimum. In summary, we have the following conditions for the global structures of the "energy potential" $\mathcal{E}_{\text {sp }}$ (see Fig. 1):

$$
\begin{array}{r}
\text { metastable: }(\lambda+\mu)^{2}+4 k(\alpha+\beta)<0, \\
\text { degenerate: }(\lambda+\mu)^{2}+4 k(\alpha+\beta)=0, \\
\text { global: }(\lambda+\mu)^{2}+4 k(\alpha+\beta)>0 .
\end{array}
$$


We now discuss the stability of the vacuum including the $\dot{\varphi}, \partial_{2} \varphi, \partial_{3} \varphi$ directions. The local stability is guaranteed by the condition $\left.\mathcal{M}\right|_{0} \geq 0$. As discussed in Sec. II B, since the "off-diagonal parts" of $\mathbf{L}^{m n}$ vanish and $\dot{\varphi}=\dot{\bar{\varphi}}=0$, the matrices $\mathbf{M}^{m n}$ are simply given by Eq. (23) and the generalized mass matrix is

$$
\left.\mathcal{M}\right|_{0}=\left(\begin{array}{cccc}
\left.\mathbf{M}^{00}\right|_{0} & & & \\
& \left.\mathbf{M}^{11}\right|_{0} & & \\
& & \left.\mathbf{M}^{22}\right|_{0} & \\
& & & \left.\mathbf{M}^{33}\right|_{0}
\end{array}\right),
$$

which is totally block diagonal. One can easily find eigenvalues of each sector. The eigenvalues of $\left.\mathbf{M}^{00}\right|_{0}$ are

$$
\begin{aligned}
& A_{1}=\frac{2(\beta-\mu) \Upsilon}{3(\alpha+\beta)}, \\
& A_{2}=\frac{12 \alpha k(\alpha+\beta)-2(3 \beta(-\beta+2 \lambda+\mu)-\alpha(3 \beta-2 \lambda+\mu)) \Upsilon}{9(\alpha+\beta)^{2}},
\end{aligned}
$$

where we have defined

$$
\Upsilon \equiv \sqrt{3 k(\alpha+\beta)+(\lambda+\mu)^{2}}+\lambda+\mu,
$$

and the eigenvalues of $\left.\mathbf{M}^{11}\right|_{0}$ are

$$
B_{1}=0, \quad B_{2}=-\frac{4(\lambda+\mu) \Upsilon}{3(\alpha+\beta)}-4 k
$$

The eigenvalues of $\left.\mathbf{M}^{22}\right|_{0}$ and $\left.\mathbf{M}^{33}\right|_{0}$ are

$C_{1}=0, \quad C_{2}=\frac{12 \alpha k(\alpha+\beta)-4(\alpha(\lambda-2 \mu)+3 \beta \lambda) \Upsilon}{9(\alpha+\beta)^{2}}$,

respectively. The eigenvalue $B_{1}=0$ corresponds to the expected zero mode. Later, we will confirm that $B_{1}=0$ is indeed the generalized NG mode and discuss the meaning of the other zero modes for $C_{1}=0$.

One finds a parameter region for which all the nonzero eigenvalues become positive. For example, if we consider $\beta=\mu=0,{ }^{6}$ the eigenvalues become

$$
\begin{aligned}
& \mathbf{M}^{00}: A_{1}=0, \quad A_{2}=\frac{12 \alpha^{2} k-4 \alpha \lambda\left(\lambda+\sqrt{3 k \alpha+\lambda^{2}}\right)}{9 \alpha^{2}}, \\
& \mathbf{M}^{11}: B_{1}=0, \quad B_{2}=-\frac{4}{3 \alpha}\left\{3 \alpha k+\lambda^{2}+\lambda \sqrt{3 \alpha k+\lambda^{2}}\right\}, \\
& \mathbf{M}^{22}=\mathbf{M}^{33}: C_{1}=0, \quad C_{2}=\frac{12 \alpha^{2} k-4 \alpha \lambda\left(\lambda+\sqrt{3 k \alpha+\lambda^{2}}\right)}{9 \alpha^{2}} .
\end{aligned}
$$

\footnotetext{
${ }^{6}$ This is also the supersymmetric limit [33].
}

One can easily show that all the nonzero eigenvalues $A_{2}, B_{2}$, $C_{2}$ are positive in the region $\alpha<0, \lambda>0$ and $\lambda^{2}+3 \alpha k>0$, and the generalized mass matrix $\left.\mathcal{M}\right|_{0}$ is positive semidefinite. Thus in this region the spatially modulated configuration (65) is a stable vacuum in the model (49).

\section{Generalized NG modes}

Next, we examine the generalized NG modes in the spatially modulated vacuum (65). The field that has a nonzero VEV is $\varphi_{1}=\partial_{1} \varphi$. The VEV is characterized by

$$
\left\langle 0\left|\varphi_{1}\right| 0\right\rangle=v_{1}=i c \varphi_{0} e^{i c x^{1}} .
$$

As discussed in Sec. II B, the relevant symmetry breaking of the spatially modulated vacuum is $U(1) \times \mathcal{P}^{1} \rightarrow$ $\left[U(1) \times \mathcal{P}^{1}\right]_{\text {diag }}$. The corresponding broken and unbroken generators are given in Ref. [32] by

$$
T_{\mathrm{b}}=P^{1}+T_{U(1)}, \quad T_{\mathrm{ub}}=P^{1}-T_{U(1)},
$$

respectively, where each generator acts on the VEV $v=i c \varphi_{0} e^{i c x^{1}}$ as

$$
\begin{array}{cl}
P^{1} v_{1}=i c \varphi_{0} c e^{i c x^{1}}, & P^{1} \bar{v}_{1}=-i c \varphi_{0} c e^{-i c x^{1}}, \\
T_{U(1)} v_{1} & =i c \varphi_{0} c e^{i c x^{1}}, \quad T_{U(1)} \bar{v}_{1}=-i c \varphi_{0} c e^{-i c x^{1}} .
\end{array}
$$

Then, the unbroken generator acts on the VEV as $T_{\mathrm{ub}} \vec{v}=0$. For the broken generator, we have

$$
T_{\mathrm{b}} \vec{v}=2 i c^{2} \varphi_{0}\left(\begin{array}{c}
e^{i c x^{1}} \\
e^{-i c x^{1}}
\end{array}\right) .
$$

On the other hand, the normalized eigenvector of $\left.\mathbf{M}^{11}\right|_{0}$ associated with $B_{1}=0$ is found to be

$$
B_{1}=0: \vec{u}_{1}=\frac{1}{\sqrt{2}}\left(\begin{array}{c}
e^{i c x^{1}} \\
e^{-i c x^{1}}
\end{array}\right) .
$$

One then finds that

$$
\vec{u}_{1} \propto T_{\mathrm{b}} \vec{v}
$$

is the generalized NG mode.

A comment on the extra zero modes $C_{1}=0$ appearing in the $i=2,3$ sectors is in store. We have chosen the $x^{1}$ direction as a specific direction of the spatial modulation. We have then found that the configuration $\left\langle\partial_{1} \varphi\right\rangle \neq 0$ is a (local) minimum of the energy density and there is a flat direction corresponding to the zero modes of $\left.\mathbf{M}^{11}\right|_{0}$. However, since the model that we consider is Lorentz invariant, the spatial directions $x^{1}, x^{2}, x^{3}$ are equivalent due to the $S O(3) \in S O(1,3)$ rotational symmetry. Therefore there should be flat directions even in the $\partial_{2} \varphi, \partial_{3} \varphi$ 
directions in the energy density. In this sense, the zero modes corresponding to $C_{1}=0$ are accompanying NG modes to the legitimate generalized NG mode in $x^{1}$ direction. On the other hand, the zero mode $A_{1}=0$ in Eq. (81) is genuinely accidental since it is nonzero for general parameters and appears only for the specific choice of parameters $\mu=\beta=0$.

\section{Lagrangian for the fluctuation modes}

We will now write down the Lagrangian for the fluctuation modes in the spatially modulated vacuum. The Lagrangian (17) becomes

$$
\mathcal{L}=\left.\frac{1}{2} \sum_{m=0}^{3} \vec{\phi}_{m}^{\dagger} \mathbf{L}^{m m}\right|_{0} \vec{\phi}_{m},
$$

where we have ignored the irrelevant constant. The eigenvalues of $\mathbf{L}^{00}$ are positive and those of $\mathbf{L}^{i i}=-\mathbf{M}^{i i}(i=1$, 2,3 ) are negative or zero in the appropriate parameter region. Therefore, the fluctuation fields have the correct signs of their canonical kinetic terms and we expect that there are no dynamical ghost modes in the Lagrangian. Since $\mathbf{M}^{00}=\mathbf{L}^{00}, \mathbf{M}^{i i}=-\mathbf{L}^{i i}$ (no summation over $i$ ), the zero modes of $\mathbf{L}^{i j}$ and $\mathbf{M}^{i j}$ coincide. We therefore conclude that the kinetic term of the generalized NG mode vanishes for the spatially modulated vacuum.

As mentioned above, the results in this subsection are essentially the same as those in Ref. [32]. However, the situation drastically changes when we consider modulations in the temporal and the lightlike directions, which we shall turn to next.

\section{Temporal modulation}

For the temporal modulation, we assume the ansatz for the VEV:

$$
\left\langle\partial_{1} \varphi\right\rangle=\left\langle\partial_{2} \varphi\right\rangle=\left\langle\partial_{3} \varphi\right\rangle=0, \quad\langle\varphi\rangle=\varphi_{0} e^{i \omega x^{0}} .
$$

with real constants $\varphi_{0}, \omega$. Assuming this configuration, the matrices $\mathbf{L}^{m n}$ can be evaluated as

$$
\begin{aligned}
& \mathbf{L}^{00}=\left(\begin{array}{cc}
k+4(\lambda+\mu)|\dot{\varphi}|^{2}-9(\alpha+\beta)|\dot{\varphi}|^{4} & 2 \dot{\varphi}^{2}\left\{-3(\alpha+\beta)|\dot{\varphi}|^{2}+(\lambda+\mu)\right\} \\
2 \dot{\bar{\varphi}}^{2}\left\{-3(\alpha+\beta)|\dot{\varphi}|^{2}+(\lambda+\mu)\right\} & k+4(\lambda+\mu)|\dot{\varphi}|^{2}-9(\alpha+\beta)|\dot{\varphi}|^{4}
\end{array}\right), \\
& \mathbf{L}^{11}=\mathbf{L}^{22}=\mathbf{L}^{33}=\left(\begin{array}{cc}
-k-2 \mu|\dot{\varphi}|^{2}+(\alpha+3 \beta)|\dot{\varphi}|^{4} & -2(\dot{\varphi})^{2}\left(\lambda-\alpha|\dot{\varphi}|^{2}\right) \\
-2(\dot{\bar{\varphi}})^{2}\left(\lambda-\alpha|\dot{\varphi}|^{2}\right) & -k-2 \mu|\dot{\varphi}|^{2}+(\alpha+3 \beta)|\dot{\varphi}|^{4}
\end{array}\right),
\end{aligned}
$$

and the others are zero. We also have the matrices

$$
\begin{aligned}
\mathbf{M}^{00}=\frac{\partial \mathbf{L}^{00}}{\partial \dot{\varphi}} \dot{\varphi}+\frac{\partial \mathbf{L}^{00}}{\partial \dot{\bar{\varphi}}} \dot{\bar{\varphi}}+\mathbf{L}^{00}= & \left(\begin{array}{cc}
k+12(\lambda+\mu)|\dot{\varphi}|^{2}-45(\alpha+\beta)|\dot{\varphi}|^{4} & 6 \dot{\varphi}^{2}\left[-5(\alpha+\beta)|\dot{\varphi}|^{2}+(\lambda+\mu)\right] \\
6 \dot{\bar{\varphi}}^{2}\left[-5(\alpha+\beta)|\dot{\varphi}|^{2}+(\lambda+\mu)\right] & k+12(\lambda+\mu)|\dot{\varphi}|^{2}-45(\alpha+\beta)|\dot{\varphi}|^{4}
\end{array}\right), \\
\mathbf{M}^{11}=\mathbf{M}^{22} & =\mathbf{M}^{33}=\frac{\partial \mathbf{L}^{11}}{\partial \dot{\varphi}} \dot{\varphi}+\frac{\partial \mathbf{L}^{11}}{\partial \dot{\bar{\varphi}}} \dot{\bar{\varphi}}-\mathbf{L}^{11} \\
& =\left(\begin{array}{cc}
3(\alpha+3 \beta)|\dot{\varphi}|^{4}-2 \mu|\dot{\varphi}|^{2}+k & 2 \dot{\varphi}^{2}\left(-\lambda+3 \alpha|\dot{\varphi}|^{2}\right) \\
2 \dot{\bar{\varphi}}^{2}\left(-\lambda+3 \alpha|\dot{\varphi}|^{2}\right) & 3(\alpha+3 \beta)|\dot{\varphi}|^{4}-2 \mu|\dot{\varphi}|^{2}+k
\end{array}\right) .
\end{aligned}
$$

Let us analyze the conditions (4), (9). Since $\frac{\partial \mathcal{L}}{\partial\left(\partial_{i} \varphi\right)}=0$ is trivially satisfied, these conditions reduce to

$$
\mathbf{L}^{00}\left(\begin{array}{c}
\ddot{\varphi} \\
\ddot{\bar{\varphi}}
\end{array}\right)=0, \quad \mathbf{L}^{00}\left(\begin{array}{c}
\dot{\varphi} \\
\dot{\bar{\varphi}}
\end{array}\right)=0
$$

Since $\ddot{\varphi}=i \omega \dot{\varphi}, \ddot{\bar{\varphi}}=-i \omega \dot{\bar{\varphi}}$, the above conditions can be rewritten as

$$
\mathbf{L}^{00}\left(\begin{array}{c}
\dot{\varphi} \\
-\dot{\bar{\varphi}}
\end{array}\right)=0, \quad \mathbf{L}^{00}\left(\begin{array}{c}
\dot{\varphi} \\
\dot{\bar{\varphi}}
\end{array}\right)=0 .
$$

Hence, they are satisfied when $\mathbf{L}^{00}=0$. This is consistent with condition (32) in the general discussion. The condition $\mathbf{L}^{00}=0$ imposes that the two independent components of $\mathbf{L}^{00}$ in Eq. (90) vanish. This is possible when the parameter $k$ is chosen as

$$
k=-\frac{(\lambda+\mu)^{2}}{3(\alpha+\beta)} .
$$

For this choice of parameters, the solution to the condition $\mathbf{L}^{00}=0$ is 


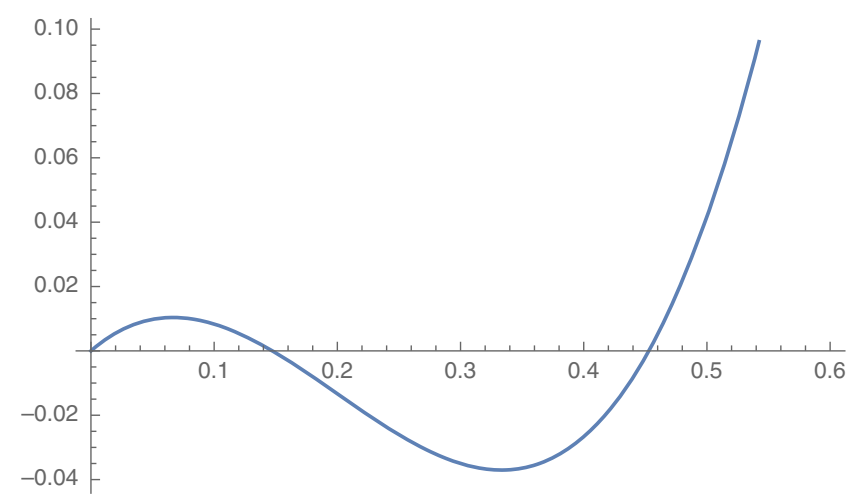

FIG. 2. The energy density as a function of $X=|\dot{\varphi}|^{2}$. The $X \neq$ 0 vacuum is the global minimum. Here $\alpha+\beta=-1, \lambda+\mu=-1$.

$$
|\dot{\varphi}|^{2}=\left|\omega \varphi_{0}\right|^{2}=\frac{\lambda+\mu}{3(\alpha+\beta)} .
$$

Note that since $k$ is the coefficient of the canonical (quadratic) kinetic term, it should be positive $k>0$. Then we find $\alpha+\beta<0$ and $\lambda+\mu<0$ are necessary for the temporal modulation.

\section{Global aspects of the potential and stability of the vacuum}

The energy density for the ansatz (89) is

$$
\mathcal{E}_{\text {temp }}=k|\dot{\varphi}|^{2}+3(\mu+\lambda)|\dot{\varphi}|^{4}-5(\alpha+\beta)|\dot{\varphi}|^{6} .
$$

This is a function of $X \equiv|\dot{\varphi}|^{2} \geq 0: \quad y=X(k+3(\mu+\lambda) X-$ $\left.5(\alpha+\beta) X^{2}\right)$. Besides the trivial vacuum $X=0$, there are nontrivial extrema. The discriminant $\mathbf{D}$ for $y^{\prime}=k+$ $6(\lambda+\mu) X-15(\alpha+\beta) X^{2}=0$ is

$$
\mathbf{D}=36(\mu+\lambda)^{2}+90(\alpha+\beta) k=16(\mu+\lambda)^{2} .
$$

This is always positive $\mathbf{D}>0$. Therefore, there is a local minimum in the $\left(X=|\dot{\varphi}|^{2}\right)$ direction, as expected. The solutions to $y^{\prime}=0$ are

$$
X=|\dot{\varphi}|^{2}=\frac{\mu+\lambda}{15(\alpha+\beta)}, \quad \frac{\mu+\lambda}{3(\alpha+\beta)} .
$$

The former is a local maximum while the latter is the temporally modulated vacuum (96). In order to clarify the (meta)stability of the temporally modulated vacuum, we examine the zeros of $f$ which is defined by $y=X f$. Since the discriminant of $f \equiv-5(\alpha+\beta) X^{2}+3(\mu+\lambda) X+k=0$ is always positive,

$\mathbf{D}_{f}=9(\mu+\lambda)^{2}+20 k(\alpha+\beta)=\frac{7}{3}(\lambda+\mu)^{2}>0$,

the vacuum (96) is energetically favored compared with the trivial vacuum $X=0$. Thus, it is the global minimum at least in the $|\dot{\varphi}|$ direction (see Fig. 2). We note that the situation is different from the spatial case. In order that the temporally modulated vacuum is allowed, the coefficient $k$ of the canonical kinetic term should be related to those of the higher-derivative terms via the relation (95) and $\alpha+\beta<0$, $\lambda+\mu<0$. Therefore, the model is severely constrained and there are no possibilities for having metastable or degenerate vacua as in the spatial modulation case.

Since $\frac{\partial \mathbf{L}^{0 i}}{\partial \dot{\varphi}}=0, \frac{\partial \mathbf{L}^{i j}}{\partial \dot{\varphi}}=0(i \neq j)$ for the ansatz (89), we have the mass matrices (91), (92). Hence, the generalized mass matrix is

$$
\left.\mathcal{M}\right|_{0}=\left(\begin{array}{cccc}
\left.\mathbf{M}^{00}\right|_{0} & & & \\
& \left.\mathbf{M}^{11}\right|_{0} & & \\
& & \left.\mathbf{M}^{22}\right|_{0} & \\
& & & \left.\mathbf{M}^{33}\right|_{0}
\end{array}\right),
$$

where $k$ is given in Eq. (95). The eigenvalues of $\mathbf{M}^{00}$ are

$$
A_{1}=0, \quad A_{2}=-\frac{8}{3} \frac{(\lambda+\mu)^{2}}{\alpha+\beta} .
$$

There is a zero mode and $A_{2}>0$ as expected. The eigenvalues of $\mathbf{M}^{11}, \mathbf{M}^{22}, \mathbf{M}^{33}$ are

$$
B_{1}=0, \quad B_{2}=\frac{4(\lambda+\mu)(\beta \lambda-\alpha \mu)}{3(\alpha+\beta)^{2}} .
$$

One can always choose the parameters so that $B_{2}>0$ by choosing $\alpha+\beta<0, \lambda+\mu<0$. $^{7}$

\section{Generalized NG modes}

The symmetry breaking pattern is essentially the same with the spatial case: $U(1) \times \mathcal{P}^{0} \rightarrow\left[U(1) \times \mathcal{P}^{0}\right]_{\text {diag }}$. The corresponding broken and unbroken generators are given by

$$
T_{\mathrm{b}}=P^{0}+T_{U(1)}, \quad T_{\mathrm{ub}}=P^{0}-T_{U(1)},
$$

respectively, where each generator acts on the VEV $v_{0}=$ $\langle\dot{\varphi}\rangle=i \omega \varphi_{0} e^{i \omega x^{0}}$ as

$$
\begin{aligned}
P^{0} v_{0} & =i \omega \varphi_{0} \omega e^{i \omega x^{0}}, \quad P^{0} \bar{v}_{0}=-i \omega \varphi_{0} \omega e^{-i \omega x^{0}}, \\
T_{U(1)} v_{0} & =i \omega \varphi_{0} \omega e^{i \omega x^{0}}, \quad T_{U(1)} \bar{v}_{0}=-i \omega \varphi_{0} \omega e^{-i \omega x^{0}} .
\end{aligned}
$$

Then, we have $T_{\mathrm{ub}} \vec{v}=0$, and

$$
T_{\mathrm{b}} \vec{v}=2 i c^{2} \varphi_{0}\left(\begin{array}{c}
e^{i \omega x^{1}} \\
e^{-i \omega x^{1}}
\end{array}\right)
$$

On the other hand, the normalized eigenvector of $\mathbf{M}^{00}$ associated with the zero eigenvalue $A_{1}=0$ is found to be

\footnotetext{
${ }^{7}$ Note that in the supersymmetric limit $\mu, \beta \rightarrow 0$, we have an extra zero mode $B_{2}=0$; see Ref. [33].
} 


$$
A_{1}=0: \vec{u}_{1}=\frac{1}{\sqrt{2}}\left(\begin{array}{c}
e^{i \omega x^{0}} \\
e^{-i \omega x^{0}}
\end{array}\right)
$$

One therefore finds that

$$
\vec{u}_{1} \propto T_{\mathrm{b}} \vec{v}
$$

is a generalized NG mode.

The zero modes corresponding to $B_{1}=0$ are accidental since the normalized eigenvectors of $\mathbf{M}^{11}=\mathbf{M}^{22}=\mathbf{M}^{33}$ are

$$
B_{1}=0: \vec{u}_{1}=\frac{1}{\sqrt{2}}\left(\begin{array}{c}
e^{i \omega x^{0}} \\
-e^{-i \omega x^{0}}
\end{array}\right)
$$

Hence, the zero modes in $\left.\mathbf{M}^{11}\right|_{0}=\left.\mathbf{M}^{22}\right|_{0}=\left.\mathbf{M}^{33}\right|_{0}$ do not correspond to eigenvectors proportional to the vector generated by the broken generator $T_{\mathrm{b}} \vec{v}$.

\section{Lagrangian for fluctuation modes}

For the temporal modulation, there are no direct correspondences between the fluctuation modes and the zero modes of $\left.\mathcal{M}\right|_{0}$, i.e., the generalized NG modes. This is because the matrices $\mathbf{L}^{m n}$ are different from the components of the generalized matrix $\mathbf{M}^{m n}$.

Since the matrix $\mathbf{L}^{00}$ vanishes in the temporally modulated vacuum, there is no canonical kinetic term for the fluctuation in the temporal direction. Only the gradient kinetic term survives in the fluctuation dynamics. The Lagrangian (17) becomes

$$
\mathcal{L}=\left.\frac{1}{2} \sum_{i=1}^{3} \vec{\phi}_{i}^{\dagger} \mathbf{L}^{i i}\right|_{0} \vec{\phi}_{i}
$$

In order to clarify whether physically unacceptable modes appear or not, we need the eigenvalues of the matrices

$$
\left.\mathbf{L}^{11}\right|_{0}=\left.\mathbf{L}^{22}\right|_{0}=\left.\mathbf{L}^{33}\right|_{0}=\left(\begin{array}{cc}
-k-2 \mu|\dot{\varphi}|^{2}+(\alpha+3 \beta)|\dot{\varphi}|^{4} & -2 \dot{\varphi}^{2}\left(\lambda-\alpha|\dot{\varphi}|^{2}\right) \\
-2 \dot{\bar{\varphi}}^{2}\left(\lambda-\alpha|\dot{\varphi}|^{2}\right) & -k-2 \mu|\dot{\varphi}|^{2}+(\alpha+3 \beta)|\dot{\varphi}|^{4}
\end{array}\right),
$$

where $k=-\frac{(\lambda+\mu)^{2}}{3(\alpha+\beta)},|\dot{\varphi}|^{2}=\frac{\lambda+\mu}{3(\alpha+\beta)}$. The eigenvalues/vectors are found to be

$$
\begin{aligned}
& s_{1}=0: \vec{u}_{1}=\frac{1}{\sqrt{2}}\left(\begin{array}{c}
e^{i \omega x^{0}} \\
-e^{-i \omega x^{0}}
\end{array}\right), \\
& s_{2}=\frac{4(\lambda+\mu)(2 \alpha \lambda-\alpha \mu+3 \beta \lambda)}{9(\alpha+\beta)^{2}}: \vec{u}_{2}=\frac{1}{\sqrt{2}}\left(\begin{array}{c}
e^{i \omega x^{0}} \\
e^{-i \omega x^{0}}
\end{array}\right) .
\end{aligned}
$$

We can always choose the parameters so that $s_{2}<0$ by obeying the conditions $\alpha+\beta<0, \lambda+\mu<0$. ${ }^{8}$ Therefore we can exclude unphysical ghost modes in appropriate regions of parameters. An alternative discussion of the fluctuations and NG modes in a time-dependent vacuum can be found in Ref. [39].

\section{Lightlike modulation}

For the lightlike modulation, we assume the VEV:

$$
\begin{aligned}
\left\langle\partial_{2} \varphi\right\rangle & =\left\langle\partial_{3} \varphi\right\rangle=0, \quad\left\langle\partial_{0} \varphi\right\rangle= \pm\left\langle\partial_{1} \varphi\right\rangle \neq 0, \\
\langle\varphi\rangle & =\varphi_{0} e^{i \omega\left(x^{0} \pm x^{1}\right)} .
\end{aligned}
$$

\footnotetext{
${ }^{8}$ For example, in the supersymmetric limit $\mu=\beta=0$, we find $s_{2}=\frac{8 \lambda^{2}}{9 \alpha}<0$ for $\alpha<0$, which corresponds to the correct sign of the gradient kinetic term.
}

This implies

$$
\left.\partial \varphi \cdot \partial \varphi\right|_{0}=\left.\partial \varphi \cdot \partial \bar{\varphi}\right|_{0}=0
$$

In the following, we can choose the + sign in Eq. (69) without loss of generality. With the ansatz (113), the matrices $\mathbf{L}^{m n}$ can be evaluated as

$$
\begin{aligned}
& \mathbf{L}^{00}=\left(\begin{array}{cc}
k+4 \lambda|\dot{\varphi}|^{2}+2 \mu|\dot{\varphi}|^{2} & 2 \mu \dot{\varphi}^{2} \\
2 \mu \dot{\bar{\varphi}}^{2} & k+4 \lambda|\dot{\varphi}|^{2}+2 \mu|\dot{\varphi}|^{2}
\end{array}\right), \\
& \mathbf{L}^{01}=\left(\begin{array}{cc}
-4 \lambda \partial_{1} \varphi \dot{\bar{\varphi}}-2 \mu \partial_{1} \bar{\varphi} \dot{\varphi} & -2 \mu \partial_{1} \varphi \dot{\varphi} \\
-2 \mu \partial_{1} \bar{\varphi} \dot{\bar{\varphi}} & -4 \lambda \partial_{1} \bar{\varphi} \dot{\varphi}-2 \mu \partial_{1} \varphi \dot{\bar{\varphi}}
\end{array}\right), \\
& \mathbf{L}^{10}=\mathbf{L}^{\dagger 01}=\left(\begin{array}{cc}
-4 \lambda \partial_{1} \bar{\varphi} \dot{\varphi}-2 \mu \partial_{1} \varphi \dot{\bar{\varphi}} & -2 \mu \partial_{1} \varphi \dot{\varphi} \\
-2 \mu \partial_{1} \bar{\varphi} \dot{\bar{\varphi}} & -4 \lambda \partial_{1} \varphi \dot{\bar{\varphi}}-2 \mu \partial_{1} \bar{\varphi} \dot{\varphi}
\end{array}\right), \\
& \mathbf{L}^{11}=\left(\begin{array}{cc}
-k+\left(\begin{array}{cc}
4 \lambda+2 \mu)\left|\partial_{1} \varphi\right|^{2} & 2 \mu\left(\partial_{1} \varphi\right)^{2} \\
2 \mu\left(\partial_{1} \bar{\varphi}\right)^{2} & -k+(4 \lambda+2 \mu)\left|\partial_{1} \varphi\right|^{2}
\end{array}\right), \\
\mathbf{L}^{22}=
\end{array}\right. \\
& \mathbf{L}^{33}=\left(\begin{array}{cc}
-k & 0 \\
0 & -k
\end{array}\right),
\end{aligned}
$$

and the others are zero. We further obtain 


$$
\begin{aligned}
& \frac{\partial \mathbf{L}^{00}}{\partial \dot{\varphi}}=\left(\begin{array}{cc}
\dot{\bar{\varphi}}\left[-8 \alpha|\dot{\varphi}|^{2}-6 \beta|\dot{\varphi}|^{2}+4(\lambda+\mu)\right] & \dot{\varphi}\left[4(\lambda+\mu)-8 \alpha|\dot{\varphi}|^{2}-6 \beta|\dot{\varphi}|^{2}\right] \\
-6 \beta \dot{\bar{\varphi}}^{3} & \dot{\bar{\varphi}}\left[-8 \alpha|\dot{\varphi}|^{2}-6 \beta|\dot{\varphi}|^{2}+4(\lambda+\mu)\right]
\end{array}\right), \\
& \frac{\partial \mathbf{L}^{01}}{\partial \dot{\varphi}}=\left(\begin{array}{cc}
4 \alpha|\dot{\varphi}|^{2} \partial_{1} \bar{\varphi}+4 \alpha \dot{\bar{\varphi}}^{2} \partial_{1} \varphi-2 \mu \partial_{1} \bar{\varphi}+6 \beta|\dot{\varphi}|^{2} \partial_{1} \bar{\varphi} & 4 \alpha|\dot{\varphi}|^{2} \partial_{1} \varphi+4 \alpha \dot{\varphi}^{2} \partial_{1} \bar{\varphi}-2 \mu \partial_{1} \varphi+6 \beta|\dot{\varphi}|^{2} \partial_{1} \varphi \\
6 \beta \dot{\bar{\varphi}}^{2} \partial_{1} \bar{\varphi} & 4 \alpha|\dot{\varphi}|^{2} \partial_{1} \bar{\varphi}+4 \alpha|\dot{\varphi}|^{2} \partial_{1} \bar{\varphi}-4 \lambda \partial_{1} \bar{\varphi}+6 \beta \dot{\bar{\varphi}}^{2} \partial_{1} \varphi
\end{array}\right), \\
& \frac{\partial \mathbf{L}^{11}}{\partial \dot{\varphi}}=\left(\begin{array}{cc}
-4 \alpha \dot{\varphi}\left(\partial_{1} \bar{\varphi}\right)^{2}-4 \alpha \dot{\bar{\varphi}}\left|\partial_{1} \varphi\right|^{2}-2 \mu \dot{\bar{\varphi}}-6 \beta \dot{\bar{\varphi}}^{2}\left|\partial_{1} \varphi\right|^{2} & -8 \alpha \dot{\varphi}\left|\partial_{1} \varphi\right|^{2}-4 \lambda \dot{\varphi}-6 \beta \dot{\bar{\varphi}}\left(\partial_{1} \varphi\right)^{2} \\
-6 \beta \dot{\bar{\varphi}}\left(\partial_{1} \bar{\varphi}\right)^{2} & -4 \alpha \dot{\varphi}\left(\partial_{1} \bar{\varphi}\right)^{2}-4 \alpha \dot{\bar{\varphi}}\left|\partial_{1} \varphi\right|^{2}-2 \mu \dot{\bar{\varphi}}-6 \beta \dot{\bar{\varphi}}\left|\partial_{1} \varphi\right|^{2}
\end{array}\right), \\
& \frac{\partial \mathbf{L}^{22}}{\partial \dot{\varphi}}=\frac{\partial \mathbf{L}^{33}}{\partial \dot{\varphi}}=\left(\begin{array}{cc}
-2 \mu \dot{\varphi} & 0 \\
-4 \lambda \dot{\bar{\varphi}} & -2 \mu \dot{\varphi}
\end{array}\right) \text {, }
\end{aligned}
$$

and the others are zero. Using these results, we can calculate

$$
\begin{aligned}
& \mathbf{M}^{00}=\frac{\partial \mathbf{L}^{00}}{\partial \dot{\varphi}} \dot{\varphi}+\frac{\partial \mathbf{L}^{00}}{\partial \dot{\bar{\varphi}}} \dot{\bar{\varphi}}+\mathbf{L}^{00}=\left(\begin{array}{cc}
k+(12 \lambda+10 \mu)|\dot{\varphi}|^{2}-(16 \alpha+12 \beta)|\dot{\varphi}|^{4} & \dot{\varphi}^{2}\left[4 \lambda+6 \mu-(8 \alpha+12 \beta)|\dot{\varphi}|^{2}\right] \\
\dot{\bar{\varphi}}^{2}\left[4 \lambda+6 \mu-(8 \alpha+12 \beta)|\dot{\varphi}|^{2}\right] & k+(12 \lambda+10 \mu)|\dot{\varphi}|^{2}-(16 \alpha+12 \beta)|\dot{\varphi}|^{4}
\end{array}\right), \\
& \mathbf{M}^{01}=\frac{\partial \mathbf{L}^{01}}{\partial \dot{\varphi}} \dot{\varphi}+\frac{\partial \mathbf{L}^{01}}{\partial \dot{\bar{\varphi}}} \dot{\bar{\varphi}}=\left(\begin{array}{cc}
-2 \mu|\dot{\varphi}|^{2}-4 \lambda|\dot{\varphi}|^{2}+4(4 \alpha+3 \beta)|\dot{\varphi}|^{4} & \dot{\varphi}^{2}\left[-2 \mu+(8 \alpha+12 \beta)|\dot{\varphi}|^{2}\right] \\
\dot{\bar{\varphi}}^{2}\left[-2 \mu+(8 \alpha+12 \beta)|\dot{\varphi}|^{2}\right] & -2 \mu|\dot{\varphi}|^{2}-4 \lambda|\dot{\varphi}|^{2}+4(4 \alpha+3 \beta)|\dot{\varphi}|^{4}
\end{array}\right), \\
& \mathbf{M}^{11}=\frac{\partial \mathbf{L}^{11}}{\partial \dot{\varphi}} \dot{\varphi}+\frac{\partial \mathbf{L}^{11}}{\partial \dot{\bar{\varphi}}} \dot{\bar{\varphi}}-\mathbf{L}^{11}=\left(\begin{array}{cc}
k-(4 \lambda+6 \mu)|\dot{\varphi}|^{2}-(16 \alpha+12 \beta)|\dot{\varphi}|^{4} & \dot{\varphi}^{2}\left[-4 \lambda-2 \mu-(8 \alpha+12 \beta)|\dot{\varphi}|^{2}\right] \\
\dot{\bar{\varphi}}^{2}\left[-4 \lambda-2 \mu-(8 \alpha+12 \beta)|\dot{\varphi}|^{2}\right] & k-(4 \lambda+6 \mu)|\dot{\varphi}|^{2}-(16 \alpha+12 \beta)|\dot{\varphi}|^{4}
\end{array}\right), \\
& \mathbf{M}^{22}=\frac{\partial \mathbf{L}^{22}}{\partial \dot{\varphi}} \dot{\varphi}+\frac{\partial \mathbf{L}^{22}}{\partial \dot{\bar{\varphi}}} \dot{\bar{\varphi}}-\mathbf{L}^{22}=\left(\begin{array}{cc}
-4 \mu|\dot{\varphi}|^{2}+k & -4 \lambda \dot{\varphi}^{2} \\
-4 \lambda \dot{\bar{\varphi}}^{2} & -4 \mu|\dot{\varphi}|^{2}+k
\end{array}\right), \\
& \mathbf{M}^{33}=\frac{\partial \mathbf{L}^{33}}{\partial \dot{\varphi}} \dot{\varphi}+\frac{\partial \mathbf{L}^{33}}{\partial \dot{\bar{\varphi}}} \dot{\bar{\varphi}}-\mathbf{L}^{33}=\left(\begin{array}{cc}
-4 \mu|\dot{\varphi}|^{2}+k & -4 \lambda \dot{\varphi}^{2} \\
-4 \lambda \dot{\bar{\varphi}}^{2} & -4 \mu|\dot{\varphi}|^{2}+k
\end{array}\right),
\end{aligned}
$$

and the others are zero.

The conditions (4), (9) reduce to

$$
\begin{gathered}
\mathbf{L}^{00}\left(\begin{array}{c}
\ddot{\varphi} \\
\ddot{\bar{\varphi}}
\end{array}\right)+\left(\mathbf{L}^{01}+\mathbf{L}^{10}\right)\left(\begin{array}{c}
\partial_{1} \dot{\varphi} \\
\partial_{1} \dot{\bar{\varphi}}
\end{array}\right)+\mathbf{L}^{11}\left(\begin{array}{c}
\partial_{1}^{2} \varphi \\
\partial_{1}^{2} \bar{\varphi}
\end{array}\right)=0 \\
\mathbf{L}^{00}\left(\begin{array}{c}
\dot{\varphi} \\
\dot{\bar{\varphi}}
\end{array}\right)=0 \\
\mathbf{L}^{10}\left(\begin{array}{c}
\dot{\varphi} \\
\dot{\bar{\varphi}}
\end{array}\right)-\left(\begin{array}{c}
\frac{\partial \mathcal{L}}{\partial\left(\partial_{1} \bar{\varphi}\right)} \\
\frac{\partial \mathcal{L}}{\partial\left(\partial_{1} \varphi\right)}
\end{array}\right)=0 .
\end{gathered}
$$

Here we have used $\frac{\partial \mathcal{L}}{\partial\left(\partial_{2} \varphi\right)}=\frac{\partial \mathcal{L}}{\partial\left(\partial_{3} \varphi\right)}=0$ by assuming the ansatz. Since we have

$$
\frac{\partial \mathcal{L}}{\partial\left(\partial_{1} \varphi\right)}=-k \partial_{1} \bar{\varphi}=0, \quad \frac{\partial \mathcal{L}}{\partial\left(\partial_{1} \bar{\varphi}\right)}=-k \partial_{1} \varphi=0
$$

the condition (120) becomes
$\left(\mathbf{L}^{10}+k \mathbf{1}_{2}\right)\left(\begin{array}{c}\dot{\varphi} \\ \dot{\bar{\varphi}}\end{array}\right)=\left(\begin{array}{c}\dot{\varphi}\left(k-4 \lambda|\dot{\varphi}|^{2}-4 \mu|\dot{\varphi}|^{2}\right) \\ \dot{\bar{\varphi}}\left(k-4 \lambda|\dot{\varphi}|^{2}-4 \mu|\dot{\varphi}|^{2}\right)\end{array}\right)=0$.

Again, by the ansatz we have $|\dot{\varphi}|^{2}=\left|\partial_{1} \varphi\right|^{2}$. Then, the condition (119) becomes

$$
\left(\begin{array}{c}
\left(k+4 \mu|\dot{\varphi}|^{2}+4 \lambda|\dot{\varphi}|^{2}\right) \dot{\varphi} \\
\left(k+4 \mu|\dot{\varphi}|^{2}+4 \lambda|\dot{\varphi}|^{2}\right) \dot{\bar{\varphi}}
\end{array}\right)=0 .
$$

These conditions are satisfied when

$$
k=\lambda+\mu=0 .
$$

For the lightlike modulation, we have $\dot{\varphi}=\partial_{1} \varphi$. Therefore, the condition (118) becomes

$$
\left(\mathbf{L}^{00}+\mathbf{L}^{01}+\mathbf{L}^{10}+\mathbf{L}^{11}\right)\left(\begin{array}{c}
\ddot{\varphi} \\
\ddot{\bar{\varphi}}
\end{array}\right)=0
$$

However, this condition is automatically satisfied since the matrix $\mathbf{L}^{00}+\mathbf{L}^{01}+\mathbf{L}^{10}+\mathbf{L}^{11}$ is identically zero by explicit calculations. Therefore, the lightlike modulation is possible when the parameters of the model satisfy Eq. (124). 
Evidently, the condition (124) is weaker than the condition $k=\lambda=\mu=0$ determined by the general discussion in section II B. The lightlike modulation requires $k=\mu+\lambda=$ 0 but $\alpha, \beta$ can be nonzero. Note that for the lightlike modulation, the amplitude $\varphi_{0}$ is not determined but is a free parameter. This is different from the spatial and the temporal modulation cases.

A comment is in order for the lightlike modulation. The model seems to be a little bit strange since the canonical kinetic term disappears in the Lagrangian (49). Although the Lagrangian contains only the fourth and the sixth order derivative terms, we will show that the dynamical (fluctuation) field has quadratic kinetic term in the Lagrangian.

\section{Global aspects of the potential and stability of the vacuum}

For the lightlike modulation, the energy density becomes constant:

$$
\mathcal{E}_{\mathrm{LL}}=0 .
$$

Therefore, there is no "potential" when we consider the ansatz (113). This is why the amplitude $\varphi_{0}$ is not determined. The generalized mass matrix is

$$
\mathcal{M}=\left(\begin{array}{ll|ll}
\mathbf{M}^{00} & \mathbf{M}^{01} & & \\
\mathbf{M}^{10} & \mathbf{M}^{11} & & \\
\hline & & \mathbf{M}^{22} & \\
& & & \mathbf{M}^{33}
\end{array}\right),
$$

where each matrix is obtained from Eq. (117) by setting $k=\mu+\lambda=0$ as

$$
\begin{aligned}
& \mathbf{M}^{00}=\mathbf{M}^{11}=-\mathbf{M}^{01}=-\mathbf{M}^{10}=\left(\begin{array}{cc}
2 \lambda|\dot{\varphi}|^{2}-(16 \alpha+12 \beta)|\dot{\varphi}|^{4} & \dot{\varphi}^{2}\left[-2 \lambda-(8 \alpha+12 \beta)|\dot{\varphi}|^{2}\right] \\
\dot{\bar{\varphi}}^{2}\left[-2 \lambda-(8 \alpha+12 \beta)|\dot{\varphi}|^{2}\right] & 2 \lambda|\dot{\varphi}|^{2}-(16 \alpha+12 \beta)|\dot{\varphi}|^{4}
\end{array}\right), \\
& \mathbf{M}^{22}=\mathbf{M}^{33}=\left(\begin{array}{cc}
4 \lambda|\dot{\varphi}|^{2} & -4 \lambda \dot{\varphi}^{2} \\
-4 \lambda \dot{\bar{\varphi}}^{2} & 4 \lambda|\dot{\varphi}|^{2}
\end{array}\right) .
\end{aligned}
$$

In contradistinction to the spatial and the temporal modulations, we have nonvanishing off-diagonal blocks $\mathbf{M}^{01}$, $\mathbf{M}^{10}$. The eigenvalues of the submatrix

$$
\tilde{M} \equiv\left(\begin{array}{ll}
\mathbf{M}^{00} & \mathbf{M}^{01} \\
\mathbf{M}^{10} & \mathbf{M}^{11}
\end{array}\right),
$$

are

$$
\begin{aligned}
& A_{1}=0, \\
& A_{2}=0, \\
& A_{3}=-48\left(\omega \varphi_{0}\right)^{4}(\alpha+\beta), \\
& A_{4}=-8\left(\omega \varphi_{0}\right)^{2}\left(2 \alpha\left(\omega \varphi_{0}\right)^{2}-\lambda\right) .
\end{aligned}
$$

The eigenvalues of the matrices $\mathbf{M}^{22}=\mathbf{M}^{33}$ are

$$
B_{1}=0, \quad B_{2}=8 \lambda\left(\omega \varphi_{0}\right)^{2} .
$$

In order that all the nonzero eigenvalues become positive, we must require that

$$
\alpha+\beta<0, \quad \lambda>0, \quad 2 \alpha \omega^{2} \varphi_{0}^{2}<\lambda .
$$

It is always possible to choose the parameters such that they satisfy the above conditions.

\section{Generalized NG modes}

For the lightlike modulation, the symmetry breaking pattern is $U(1) \times \mathcal{P}^{0} \times \mathcal{P}^{1} \rightarrow\left[U(1) \times \mathcal{P}^{ \pm}\right]_{\text {diag }}$ where $\mathcal{P}^{ \pm}$ represent the translational symmetry group along the lightcone directions $x^{ \pm}=x^{0} \pm x^{1}$. The corresponding broken and unbroken generators are given by

$$
T_{\mathrm{b}}=P^{ \pm}+T_{U(1)}, \quad T_{\mathrm{ub}}=P^{ \pm}-T_{U(1)},
$$

respectively. In the following, we choose the + sign in $P_{ \pm}$. The actions of these generators on the VEV $\langle\dot{\varphi}\rangle=\left\langle\partial_{1} \varphi\right\rangle=$ $v_{\mathrm{LL}}=i \omega \varphi_{0} e^{i \omega\left(x^{0}+x^{1}\right)}$ are given by

$$
\begin{aligned}
P^{ \pm} v_{\mathrm{LL}} & =i \omega \varphi_{0} \omega e^{i \omega\left(x^{0}+x^{1}\right)}, \quad P^{ \pm} \bar{v}_{\mathrm{LL}}=-i \omega \varphi_{0} \omega e^{-i \omega\left(x^{0}+x^{1}\right)}, \\
T_{U(1)} v_{\mathrm{LL}} & =i \omega \varphi_{0} \omega e^{i \omega\left(x^{0}+x^{1}\right)}, \\
T_{U(1)} \bar{v}_{\mathrm{LL}} & =-i \omega \varphi_{0} \omega e^{-i \omega\left(x^{0}+x^{1}\right)} .
\end{aligned}
$$

Thus, we find $T_{\mathrm{ub}} \vec{v}=0$ and

$$
T_{\mathrm{b}} \vec{v}=2 i \omega^{2} \varphi_{0}\left(\begin{array}{c}
e^{i \omega\left(x^{0}+x^{1}\right)} \\
e^{-i \omega\left(x^{0}+x^{1}\right)} \\
e^{i \omega\left(x^{0}+x^{1}\right)} \\
e^{-i \omega\left(x^{0}+x^{1}\right)}
\end{array}\right)
$$


On the other hand, the zero eigenvalues $A_{1}=A_{2}=0$ of $\widetilde{\mathcal{M}}$ are degenerate and whose eigenvectors are

$A_{1}=0: \vec{u}_{1}=\left(\begin{array}{l}0 \\ 1 \\ 0 \\ 1\end{array}\right), \quad A_{2}=0: \vec{u}_{2}=\left(\begin{array}{l}1 \\ 0 \\ 1 \\ 0\end{array}\right)$.

It is clear that the following linear combination of $\vec{u}_{1}$ and $\vec{u}_{2}$ defines a zero mode of $\widetilde{\mathcal{M}}$ :

$\vec{u}_{12} \equiv \frac{1}{2}\left(e^{-i \omega\left(x^{0}+x^{1}\right)} \vec{u}_{1}+e^{i \omega\left(x^{0}+x^{1}\right)} \vec{u}_{2}\right)=\left(\begin{array}{c}e^{i \omega\left(x^{0}+x^{1}\right)} \\ e^{-i \omega\left(x^{0}+x^{1}\right)} \\ e^{i \omega\left(x^{0}+x^{1}\right)} \\ e^{-i \omega\left(x^{0}+x^{1}\right)}\end{array}\right)$.

Hence, we find the generalized NG mode is a zero mode of $\tilde{M}$ :

$$
\vec{u}_{12} \propto T_{\mathrm{b}} \vec{v}_{\mathrm{LL}}
$$

One finds that the zero modes corresponding to $B_{1}=0$ are not proportional to the vector $T_{\mathrm{b}} \vec{v}$ and thus they are accidental zero modes.

\section{Lagrangian for fluctuation modes}

For the lightlike case, since we have $\left.\mathbf{L}^{22}\right|_{0}=\left.\mathbf{L}^{33}\right|_{0}=0$, there are no gradient kinetic terms of the fluctuation in the $m=2,3$ directions. Instead, we have a cross term in the temporal and the remaining spatial directions. The Lagrangian (17) becomes

$$
\mathcal{L}=\left.\frac{1}{2} \sum_{i, j=0,1} \vec{\phi}_{i}^{\dagger} \mathbf{L}^{i j}\right|_{0} \vec{\phi}_{j}
$$

We calculate the eigenvalues of the following matrix:

$$
\tilde{\mathbf{L}} \equiv\left(\begin{array}{cc}
\left.\mathbf{L}^{00}\right|_{0} & \left.\mathbf{L}^{01}\right|_{0} \\
\left.\mathbf{L}^{10}\right|_{0} & \left.\mathbf{L}^{11}\right|_{0}
\end{array}\right)
$$

where

$$
\begin{aligned}
& \left.\mathbf{L}^{00}\right|_{0}=\left.\mathbf{L}^{11}\right|_{0}=2 \lambda\left(\begin{array}{cc}
|\dot{\varphi}|^{2} & \dot{\varphi}^{2} \\
\dot{\bar{\varphi}}^{2} & |\dot{\varphi}|^{2}
\end{array}\right), \\
& \left.\mathbf{L}^{01}\right|_{0}=\left.\mathbf{L}^{10}\right|_{0}=-\left.\mathbf{L}^{00}\right|_{0} .
\end{aligned}
$$

The eigenvalues of $\tilde{\mathbf{L}}$ are

$$
s_{1}=s_{2}=s_{3}=0, \quad s_{4}=8 \lambda\left(\omega \varphi_{0}\right)^{2} .
$$

Here we have $\omega \varphi_{0}=|\dot{\varphi}|$. Since $\lambda>0$, we have $s_{4}>0$. There is only one fluctuation mode whose kinetic term has a positive coefficient. It is, however, not obvious whether the mode is physical or unphysical. We can say that it is not an unstable mode at least in the parameter region given by Eq. (132).

\section{SUMMARY AND DISCUSSION}

In this paper, we have investigated modulated vacua in Lorentz-invariant scalar field theories. Although, there are a lot of studies on space- and time-dependent vacua based on specific models of interest in the literature, we have here performed a comprehensive analysis on spatial, temporal and lightlike modulated vacua in generic scalar field theories with appropriate properties. In particular, our models are in some sense minimal (i.e., need only sixth order in derivatives) and are globally stable. We have found the general conditions for modulated vacua. We also have discussed a generalization of the NG theorem and have clarified the relation between the zero modes of the generalized mass matrix and the generalized NG modes. According to the spontaneous symmetry breaking patterns, the modulated vacua have been classified into the spatial, temporal and lightlike ones. For the spatial and temporal modulations, the diagonal component of the $U(1)$ and translational symmetries along the $x^{1}$ or $x^{0}$ direction are preserved. For the lightlike case, the diagonal component of the $U(1)$ and the translation along the light-cone direction is preserved. We have presented the general procedure to analyze each modulated vacuum. We have then demonstrated these modulated vacua in a specific model where up to sixth-order derivative terms of a complex scalar field are included. The model is a generalization of the one considered in Ref. [32] where the spatially modulated vacuum was analyzed. We have shown that there are models that allow for temporal and lightlike modulated vacua. The global structure of the energy potential in the temporal case is severely restricted, namely, the temporally modulated state is a global vacuum at least in the $|\dot{\varphi}|$ direction. This is different from the spatially modulated case, for which there is a parameter region where the vacuum is metastable, degenerate or global minimum. We have found that the lightlike modulation is allowed in the model where only the quartic and sextic terms remain. We have then identified the generalized NG modes in each modulation. We have also written down the Lagrangians for the fluctuation modes in each modulated vacuum. As discussed in the previous paper [32], the kinetic terms of the generalized NG modes disappear in the spatial modulation. The absence of ghosts is guaranteed due to the positive semidefiniteness of the generalized mass matrix $\mathcal{M}$. In the temporal case, the kinetic term of the fluctuation along the time direction vanishes, but this does not correspond to the generalized NG mode in general. We have found that there is a possible parameter choice for 
which no ghosts occur in the temporal modulation. For the lightlike case, the gradient kinetic term along the $x^{2}, x^{3}$ directions are absent while there is only one mode that has a nonzero kinetic term in the $x^{0}, x^{1}$ directions. There are no obvious conditions for the absence of ghosts. However, we have pointed out that there is a parameter region where no ghosts appear in the fluctuation.

In this paper, we have considered only the FF-type modulation, i.e., the modulation of the phase of fields. It would be interesting to study a possibility of the LO-type where the amplitude of the fields is modulated. This could be realized by including a potential term. We have studied only the quadratic order of the fluctuations. Even though, part of the fluctuation modes are absent in each modulated vacuum, there are possible kinetic terms at higher orders. It would be interesting to study the dynamics based on these modes.

The modulated vacua in a Lorentz-invariant setup studied in this paper are relatively overlooked possibilities of vacuum structures. As an application of the present discussions, we will consider a supersymmetric extension of the present model, which is possible for $\beta=\mu=0$, and will investigate supersymmetry breaking in modulated vacua $[33,41]$. To this end, we can embed our model in higher-derivative chiral models formulated in Refs. [42-44]. A possibility to use such supersymmetry breaking vacua as a hidden sector for phenomenological models is an interesting future direction.

A generalization of the NG theorem in the classical regime has been discussed in this paper. It would also be important to investigate the quantum nature of the generalized NG modes.

\section{ACKNOWLEDGMENTS}

This work is supported by the Ministry of Education, Culture, Sports, Science (MEXT)-Supported Program for the Strategic Research Foundation at Private Universities 'Topological Science' (Grant No. S1511006). The work of M. N. is also supported in part by the Japan Society for the Promotion of Science (JSPS) Grant-in-Aid for Scientific Research (KAKENHI Grant No. 16H03984 and No. 18H01217). The work of M. N. and S. B. G. is also supported in part by a Grant-in-Aid for Scientific Research on Innovative Areas "Topological Materials Science" (KAKENHI Grant No. 15H05855) from the MEXT of Japan. The work of S. S. is supported by JSPS KAKENHI Grant No. JP17K14294. The work of R. Y. is also supported in part by a Grant-in-Aid for Scientific Research on Innovative Areas "Discrete Geometric Analysis for 859 Materials Design" (KAKENHI Grant No. 17H06462) from the MEXT of Japan.
[1] P. Fulde and R. A. Ferrell, Superconductivity in a strong spin-exchange field, Phys. Rev. 135, A550 (1964).

[2] A. I. Larkin and Y. N. Ovchinnikov, Nonuniform state of superconductors, Zh. Eksp. Teor. Fiz. 47, 1136 (1964) [Sov. Phys. JETP 20, 762 (1965)].

[3] K. Machida and H. Nakanishi, Superconductivity under a ferromagnetic molecular field, Phys. Rev. B 30, 122 (1984).

[4] R. Yoshii, S. Tsuchiya, G. Marmorini, and M. Nitta, Spin imbalance effect on Larkin-Ovchinnikov-Fulde-Ferrel state, Phys. Rev. B 84, 024503 (2011).

[5] R. Yoshii, S. Takada, S. Tsuchiya, G. Marmorini, H. Hayakawa, and M. Nitta, Fulde-Ferrell-Larkin-Ovchinnikov states in a superconducting ring with magnetic fields: Phase diagram and the first-order phase transitions, Phys. Rev. B 92, 224512 (2015).

[6] S. Brazovskii, Solitons and their arrays: From Quasi onedimensional conductors to stripes, arXiv:0709.2296.

[7] L. Radzihovsky and D. E. Sheehy, Imbalanced Feshbachresonant fermi gases, Rep. Prog. Phys. 73, 076501 (2010).

[8] L. Radzihovsky, Fluctuations and phase transitions in Larkin-Ovchinnikov liquid crystal states of populationimbalanced resonant Fermi gas, Phys. Rev. A 84, 023611 (2011).

[9] Y. Liao, A. S. C. Rittner, T. Paprotta, W. Li, G. B. Partridge, R. G. Hulet, S. K. Baur, and E. J. Mueller, Spin-imbalance in a one-dimensional Fermi gas, Nature (London) 467, 567 (2010).

[10] E. Nakano and T. Tatsumi, Chiral symmetry and density wave in quark matter, Phys. Rev. D 71, 114006 (2005).

[11] S. Karasawa and T. Tatsumi, Variational approach to the inhomogeneous chiral phase in quark matter, Phys. Rev. D 92, 116004 (2015).

[12] D. Nickel, How Many Phases Meet at the Chiral Critical Point?, Phys. Rev. Lett. 103, 072301 (2009).

[13] D. Nickel, Inhomogeneous phases in the NambuJona-Lasino and quark-meson model, Phys. Rev. D 80, 074025 (2009).

[14] G. Basar and G. V. Dunne, Self-Consistent Crystalline Condensate in Chiral Gross-Neveu and Bogoliubov-de Gennes Systems, Phys. Rev. Lett. 100, 200404 (2008).

[15] G. Basar and G. V. Dunne, A twisted kink crystal in the chiral gross-Neveu model, Phys. Rev. D 78, 065022 (2008).

[16] G. Basar, G. V. Dunne, and M. Thies, Inhomogeneous condensates in the thermodynamics of the chiral NJL(2) model, Phys. Rev. D 79, 105012 (2009).

[17] S. Nakamura, H. Ooguri, and C. S. Park, Gravity dual of spatially modulated phase, Phys. Rev. D 81, 044018 (2010).

[18] A. Amoretti, D. Aren, R. Argurio, D. Musso, and L. A. Pando Zayas, A holographic perspective on phonons and pseudo-phonons, J. High Energy Phys. 05 (2017) 051. 
[19] A. Amoretti, D. Aren, B. Goutraux, and D. Musso, DC Resistivity of Quantum Critical, Charge Density Wave States from Gauge-Gravity Duality, Phys. Rev. Lett. 120, 171603 (2018).

[20] A. Amoretti, D. Aren, B. Goutraux, and D. Musso, Effective holographic theory of charge density waves, Phys. Rev. D 97, 086017 (2018).

[21] T. Andrade and A. Krikun, Commensurate lock-in in holographic non-homogeneous lattices, J. High Energy Phys. 03 (2017) 168.

[22] R. G. Cai, L. Li, Y. Q. Wang, and J. Zaanen, Intertwined Order and Holography: The Case of Parity Breaking Pair Density Waves, Phys. Rev. Lett. 119, 181601 (2017).

[23] A. Shapere and F. Wilczek, Classical Time Crystals, Phys. Rev. Lett. 109, 160402 (2012).

[24] F. Wilczek, Quantum Time Crystals, Phys. Rev. Lett. 109, 160401 (2012).

[25] H. Watanabe and M. Oshikawa, Absence of Quantum Time Crystals, Phys. Rev. Lett. 114, 251603 (2015).

[26] J. Zhang et al., Observation of a discrete time crystal, Nature (London) 543, 217 (2017).

[27] S. Choi et al., Observation of discrete time-crystalline order in a disordered dipolar many-body system, Nature (London) 543, 221 (2017).

[28] T. Hayata and Y. Hidaka, Diffusive Nambu-Goldstone modes in quantum time-crystals, arXiv:1808.07636.

[29] T. Hayata, Y. Hidaka, and A. Yamamoto, Temporal chiral spiral in QCD in the presence of strong magnetic fields, Phys. Rev. D 89, 085011 (2014).

[30] A. Nicolis, R. Penco, F. Piazza, and R. A. Rosen, More on gapped Goldstones at finite density: More gapped Goldstones, J. High Energy Phys. 11 (2013) 055.

[31] N. Arkani-Hamed, H. C. Cheng, M. A. Luty, and S. Mukohyama, Ghost condensation and a consistent infrared modification of gravity, J. High Energy Phys. 05 (2004) 074.
[32] M. Nitta, S. Sasaki, and R. Yokokura, Spatially modulated vacua in a Lorentz-invariant scalar field theory, Eur. Phys. J. C 78, 754 (2018).

[33] M. Nitta, S. Sasaki, and R. Yokokura, Supersymmetry breaking in spatially modulated vacua, Phys. Rev. D 96, 105022 (2017).

[34] M. Ostrogradsky, Memoires sur les equations differentielles relatives au probleme des isoperimetres, Mem. Acad. St. Petersbourg 6, 385 (1850).

[35] R. P. Woodard, Avoiding dark energy with 1/r modifications of gravity, Lect. Notes Phys. 720, 403 (2007).

[36] T. G. Lee, E. Nakano, Y. Tsue, T. Tatsumi, and B. Friman, Landau-Peierls instability in a Fulde-Ferrell type inhomogeneous chiral condensed phase, Phys. Rev. D 92, 034024 (2015).

[37] Y. Hidaka, K. Kamikado, T. Kanazawa, and T. Noumi, Phonons, pions and quasi-long-range order in spatially modulated chiral condensates, Phys. Rev. D 92, 034003 (2015).

[38] D. Musso, Simplest phonons and pseudo-phonons in field theory, arXiv:1810.01799.

[39] A. Nicolis and F. Piazza, Spontaneous symmetry probing, J. High Energy Phys. 06 (2012) 025.

[40] I. Low and A. V. Manohar, Spontaneously Broken SpaceTime Symmetries and Goldstone's Theorem, Phys. Rev. Lett. 88, 101602 (2002).

[41] S. B. Gudnason, M. Nitta, S. Sasaki, and R. Yokokura, following paper, Supersymmetry breaking and ghost goldstino in modulated vacua, Phys. Rev. D 99, 045012 (2019).

[42] M. Nitta and S. Sasaki, Higher derivative corrections to manifestly supersymmetric nonlinear realizations, Phys. Rev. D 90, 105002 (2014).

[43] M. Nitta and S. Sasaki, BPS states in supersymmetric chiral models with higher derivative terms, Phys. Rev. D 90, 105001 (2014).

[44] M. Nitta and S. Sasaki, Classifying BPS states in supersymmetric gauge theories coupled to higher derivative chiral models, Phys. Rev. D 91, 125025 (2015). 